\title{
Monodromy, differential equations and the Jacobian conjecture
}

\author{
by Shmuel Friedland (Chicago)
}

\begin{abstract}
We study certain problems on polynomial mappings related to the Jacobian conjecture.

0. Introduction. Let $F: \mathbb{C}^{n} \rightarrow \mathbb{C}^{n}, n>1$, be a local polynomial diffeomorphism. The Jacobian conjecture claims that $F$ is a diffeomorphism. See [B-C-W], [Dru] and [Ess] for the surveys on this problem. It seems that the solution of the Jacobian conjecture is still out of reach. The Jacobian conjecture is on Smale's list of the problems for the next century [Sma]. The object of this paper is to study certain problems on polynomial mappings which seem to be closely related to the Jacobian conjecture and are of independent interest.

We now summarize the main results of our paper. Our main object of study is a primitive polynomial $f \in \mathbb{C}\left[\mathbb{C}^{2}\right]$. First we study the monodromy action on the regular affine fiber $V_{t}:=f^{-1}(t) \subset \mathbb{C}^{2}$. There are two main invariants here: $\operatorname{fix}_{1}(f), \mathrm{fix}^{1}(f)$, which are the dimensions of the subspaces of fixed elements in homology and cohomology, $H_{1}\left(V_{t}, \mathbb{C}\right)$ and $H^{1}\left(V_{t}, \mathbb{C}\right)$ respectively, under the action of monodromy. It turns out that $\operatorname{fix}^{1}(f)$ is equal to the invariant introduced by Stein [Ste1]: Let $\delta(f, t)$ be the number of irreducible components of $f=t$ minus one. Then

$$
\delta(f):=\sum_{t \in \mathbb{C}} \delta(f, t)
$$

Then $\operatorname{fix}^{1}(f)=\delta(f)$. We show that in general fix $x_{1}(f) \neq \mathrm{fix}^{1}(f)$. This shows that the monodromy action on the first homology (cohomology) of $V_{t}$ is not semisimple. This contrasts with Deligne's result that the monodromy action on the first homology (cohomology) of $\Sigma_{t}$, the closure of the fiber $V_{t}$, is semisimple [Del2].
\end{abstract}

1991 Mathematics Subject Classification: Primary 14D05, 14E07, 14E09.

Key words and phrases: Gauss-Manin connection, Jacobian conjecture, monodromy. 
Let $\mathcal{F}:=\mathbb{C}(f)$ be the field of rational functions in a variable $f$ and $\mathcal{F}\left[\mathbb{C}^{2}\right]:=\mathbb{C}\left[\mathbb{C}^{2}\right] \otimes \mathcal{F} \subset \mathbb{C}\left(\mathbb{C}^{2}\right)$ be the ring of polynomials in $x, y$ with coefficients in $\mathcal{F}$. Let $\operatorname{CVV}(f) \subset \mathbb{C}$ be the finite set of singular fibers $V_{t}$ and $B:=\mathbb{C} \backslash \operatorname{CVV}(f)$ be the set of regular fibers. Then $Z:=f^{-1}(B) \rightarrow B$ is a fiber bundle. Let $E \rightarrow B$ be a holomorphic vector bundle with fiber $\mathcal{H}^{1}\left(V_{t}\right)$. Here $\mathcal{H}^{1}\left(V_{t}\right)$ is the Grothendieck-de Rham cohomology of all closed rational 1-forms on $\Sigma_{t}$ which are holomorphic on $V_{t}$ modulo the exact forms. We assume that $E$ is equipped with the Gauss-Manin connection. Let $\mathcal{R}(E)$ be the set of rational sections on $E$ which are induced by rational 1-forms on $\mathbb{C}^{2}$. Then $\mathcal{R}(E)$ is a vector space over $\mathcal{F}$ of dimension $N:=\operatorname{dim} \mathcal{H}^{1}\left(V_{t}\right)=\operatorname{dim} H^{1}\left(V_{t}, \mathbb{C}\right)$.

Associate with $f$ the differential operator

$$
L:=-\frac{\partial f}{\partial y} \frac{\partial}{\partial x}+\frac{\partial f}{\partial x} \frac{\partial}{\partial y} .
$$

As $L(f)=0$ it follows that $L: \mathcal{F}\left[\mathbb{C}^{2}\right] \rightarrow \mathcal{F}\left[\mathbb{C}^{2}\right]$ is an $\mathcal{F}$-linear operator. We show that $L$ is Fredholm: $\operatorname{dim} \operatorname{ker} L=1$ and $\operatorname{dim} \operatorname{coker} L=N$. There is a natural isomorphism between $\mathcal{U}:=\mathcal{F}\left[\mathbb{C}^{2}\right] / L\left(\mathcal{F}\left[\mathbb{C}^{2}\right]\right)$ and $\mathcal{R}(E)$.

A pair $F=(f, g): \mathbb{C}^{2} \rightarrow \mathbb{C}^{2}$ is called a Jacobian pair if $\operatorname{det} J(F)=1$ everywhere and $F$ is not a diffeomorphism. If Jacobian pairs exist, contrary to the Jacobian conjecture, then they have very remarkable properties that we list. We mention the following two properties: Assume that $F$ is a Jacobian pair and let

$$
M:=\frac{\partial g}{\partial y} \frac{\partial}{\partial x}-\frac{\partial g}{\partial x} \frac{\partial}{\partial y} .
$$

Then $L M=M L$ and $L(g)=M(f)=1$. It turns out that $M$ acts on $\mathcal{U}$ as a derivation on $\mathcal{R}(E)$ with respect to the Gauss-Manin connection. A minimal resolution of $F$ yields a proper map $\check{F}: X \rightarrow \mathbb{C}^{2}$ where $X$ is an affine smooth variety in $\mathbb{C}^{n}$. It is known [For2] that $X$ embeds properly holomorphically into $\mathbb{C}^{4}$. Furthermore, $X$ embeds into $\mathbb{C}^{3}$ iff $X$ is parallelizable (as a complex manifold) [For1]. We show that $X$ is not parallelizable.

We briefly summarize the contents of our paper. $\S 1$ deals with some basic notions of the primitive polynomial $f \in \mathbb{C}\left[\mathbb{C}^{2}\right]$, the properties of its fibers $V_{t}$, and the minimal resolution $\tilde{f}: M \rightarrow \mathbb{C P}$. We also discuss the minimal resolution of a dominating polynomial map $F=(f, g): \mathbb{C}^{2} \rightarrow \mathbb{C}^{2}$. In $\S 2$ we discuss the monodromy on the first homology and cohomology of the regular fiber $V_{t}$. We prove the equality $\delta(f)=$ fix $^{1}(f)$ and discuss Deligne's example. In $\S 3$ we discuss 1 -forms on $V_{t}$ and their extensions to 1-forms on $\mathbb{C}^{2} . \S 4$ deals with the operator $L$. In $\S 5$ we discuss the Jacobian pairs.

This paper evolved through many years and various preprints. Some of the results presented here were given in [Fri]. I would like to thank all my colleagues who helped me to understand the various concepts discussed here. 
After this paper was submitted to Annales Polonici Mathematici I learned about the following results: Theorem (2.7) is a special case $(n=2)$ of Theorem 2 of Artal-Bartolo, Cassou-Noguès and Dimca [A-C-D]. A different treatment of the non-semisimplicity of monodromy (Proposition (2.9)) is given by Bailly-Maitre [Bai]. Theorem 1 of Dimca [Dim] gives a different version of Theorem (4.4) for $n=2$ and Corollary (4.5).

1. Preliminary results. Let $\Sigma$ be an oriented compact Riemannian surface $\Sigma$ of genus gen. The first homology group $H_{1}(\Sigma, \mathbb{Z})$ has an intersection product $[\alpha] \cdot[\beta]$ for any two closed curves $\alpha, \beta \subset \Sigma$. The product · : $H_{1}(\Sigma, \mathbb{Z}) \times H_{1}(\Sigma, \mathbb{Z}) \rightarrow \mathbb{Z}$ is a skew symmetric bilinear form. A set of closed simple smooth curves $\gamma_{1}, \ldots, \gamma_{2}$ gen $\subset \Sigma$ is a canonical basis in $H_{1}(\Sigma, \mathbb{Z})$ if the following conditions hold: $\gamma_{i}$ intersects $\gamma_{\mathrm{gen}+i}$ in one point and the intersection is transversal. Moreover, $\left[\gamma_{i}\right] \cdot\left[\gamma_{\text {gen }+i}\right]=1, i=1, \ldots$, gen. The intersection of $\gamma_{i}$ and $\gamma_{j}$ for $|i-j| \neq 0$, gen is empty. Hence $\left[\gamma_{i}\right] \cdot\left[\gamma_{j}\right]=0$, $|i-j| \neq$ gen. Let $J=\left(\left[\gamma_{i}\right] \cdot\left[\gamma_{j}\right]\right)_{i, j=1}^{2 n}$ be the skew symmetric matrix induced by a canonical basis in $H_{1}(\Sigma, \mathbb{Z})$. Then the transition matrix between two canonical bases in $H_{1}(\Sigma, \mathbb{Z})$ is given by an integer-valued symplectic matrix from

$$
\mathrm{Sp}(\text { gen, } \mathbb{Z}):=\left\{A \in \mathrm{SL}(2 \text { gen, } \mathbb{Z}): A^{T} J A=J\right\} .
$$

The cohomology group $H^{1}(\Sigma, \mathbb{Z})$, i.e. the integer-valued linear functionals on $H_{1}(\Sigma, \mathbb{Z})$, can be identified with $H_{1}(\Sigma, \mathbb{Z})$ using the intersection product. Let

$$
H_{1}(\Sigma, \mathbb{F})=H_{1}(\Sigma, \mathbb{Z}) \otimes \mathbb{F}, \quad H^{1}(\Sigma, \mathbb{F})=H^{1}(\Sigma, \mathbb{Z}) \otimes \mathbb{F},
$$

be the first homology and cohomology of $\Sigma$ over the field $\mathbb{F}=\mathbb{Q}, \mathbb{R}$ or $\mathbb{C}$.

Assume that $\Sigma$ is endowed with a Riemannian metric $d s^{2}$. A classical result claims that $H^{1}(\Sigma, \mathbb{R})$ is represented by 2 gen harmonic 1 -forms $\omega_{1}, \ldots, \omega_{2 \text { gen }}$ such that

$$
\int_{\gamma_{i}} \omega_{j}=\delta_{i j}, \quad i, j=1, \ldots, 2 \text { gen } .
$$

The metric $d s^{2}$ induces a complex structure on $\Sigma$. There exist gen linearly independent holomorphic 1 -forms $v_{1}, \ldots, v_{\text {gen }}$ whose real and imaginary parts are 2 gen linearly independent harmonic 1 -forms. One can normalize $v_{1}, \ldots, v_{\text {gen }}$ by the conditions

$$
\int_{\gamma_{i}} v_{j}=\delta_{i j}, \quad i, j=1, \ldots, \text { gen } .
$$

Then the Riemann matrix $R:=\left(\int_{\gamma_{\mathrm{gen}+i}} v_{j}\right)_{i, j=1}^{\text {gen }}$ is a symmetric matrix with a positive definite imaginary part $\Im R>0$ (see e.g. [F-K]). Let $\mathrm{SH}_{n}$ be the Siegel upper half plane of all $n \times n$ complex symmetric matrices with a 
positive definite imaginary part. Then $\operatorname{Sp}(n, \mathbb{Z})$ acts properly discontinuously on $\mathrm{SH}_{n}$. As the Riemann matrix $R$ is determined by the choice of a canonical homology basis in $\Sigma$ we denote by $[R(\Sigma)]$ the unique point in $\mathrm{SH}_{n} / \operatorname{Sp}(n, \mathbb{Z})$ determined by all Riemann matrices corresponding to $\Sigma$ and the complex structure $\left(\Sigma, d s^{2}\right)$. Conversely, $[R(\Sigma)]$ determines the complex structure of $\left(\Sigma, d s^{2}\right)$ (up to a biholomorphism). (See for example [Nag].)

Let $\Sigma$ be a compact Riemann surface and assume that $\zeta_{1}, \ldots, \zeta_{k} \in \Sigma$. Let $V=\Sigma \backslash\left\{\zeta_{1}, \ldots, \zeta_{k}\right\}$ be the Riemann surface punctured at $k \geq 1$ points. Let $\gamma_{2}$ gen $+i \subset V$ be a simple smooth curve bounding a simply connected domain in $\Sigma$ which contains only the point $\zeta_{i}$ out of the $k$ points $\left\{\zeta_{1}, \ldots, \zeta_{k}\right\}$ for $i=1, \ldots, k$. We shall assume that $\gamma_{2 \text { gen }+i}$ is oriented positively with respect to $\zeta_{i}$. Then $\left[\gamma_{j}\right], j=1, \ldots, 2$ gen $+k-1$, form a basis for $H_{1}(V, \mathbb{Z})$. Let $H_{1}(V, \mathbb{F})$ and $H^{1}(V, \mathbb{F})$ denote the first homology and cohomology of $V$ with coefficients in $\mathbb{F}$. Let $\mathcal{O}_{\mathrm{r}}(V)$ and $\Omega_{\mathrm{r}}^{1}(V)$ be the sets of holomorphic functions and holomorphic 1-forms (differentials) on $V$ whose singularities on $\Sigma \backslash V$ are (at most) poles. Let $\mathcal{H}^{1}(V):=\Omega_{\mathrm{r}}^{1}(V) / d \mathcal{O}_{\mathrm{r}}(V)$ be the Grothendieck-de Rham cohomology of $V$. With each $[\omega] \in H_{1}(V, \mathbb{Z})$ associate the following linear functional:

$$
[\omega]: \Omega_{\mathrm{r}}^{1}(V) \rightarrow \mathbb{C}, \quad[\omega](\varrho)=\int_{\omega} \varrho .
$$

The residue theorem $[\mathrm{F}-\mathrm{K}, \S 2.5]$ yields

$$
\sum_{i=2 \operatorname{gen}+1}^{k} \int_{\gamma_{i}} \varrho=0, \quad \varrho \in \Omega_{\mathrm{r}}^{1}(V) .
$$

Assume that $[\omega](\varrho)=0$ for all $\omega \in H_{1}(V, \mathbb{Z})$. By integrating $\varrho$ from a fixed point $\zeta_{0} \in V$ we obtain $f \in \mathcal{O}_{\mathrm{r}}(V)$ such that $\varrho=d f$. Thus $H_{1}(V, \mathbb{Z})$ is a set of linear functionals on $\mathcal{H}^{1}(V)$. Grothendieck's theorem [Gro, Thm. 1] yields

$$
N:=\operatorname{dim}\left(\mathcal{H}^{1}(V)\right)=2 \text { gen }+k-1 .
$$

Hence $H^{1}(V, \mathbb{C})$ is isomorphic to $\mathcal{H}^{1}(V)$. Thus $\left[\gamma_{i}\right], i=1, \ldots, N$, give rise to $N$ linearly independent functionals on $\mathcal{H}^{1}(V)$. Therefore there exist $N$ linearly independent holomorphic 1-forms $\varrho_{j}$ on $V$ satisfying the condition

$$
\int_{\gamma_{i}} \varrho_{j}=\delta_{i j}, \quad i, j=1, \ldots, N .
$$

Let $V \subset \mathbb{C}^{n}$ be an irreducible smooth affine curve. Then $V$ is a compact Riemann surface $\Sigma$ punctured at $k$ points $\zeta_{1}, \ldots, \zeta_{k} \in \Sigma$ for some $k \geq 1$. Note that $V$ and hence $\Sigma$ are equipped with the complex structure. As $V$ is smooth, it is well known that $\mathcal{O}_{\mathrm{r}}(V)=\mathbb{C}[V]$.

Let $f \in \mathbb{C}\left[\mathbb{C}^{2}\right]$ be a nonconstant polynomial. Consider the polynomial map $f: \mathbb{C}^{2} \rightarrow \mathbb{C}$. Extend this map to the rational map $\widehat{f}: \mathbb{C P}^{2} \rightarrow \mathbb{C P}$. 
This map has a finite number of indeterminacy points. Blow up $\mathbb{C P}^{2}$ at the indeterminacy points to obtain a complex surface $M$ so that $\widehat{f}$ lifts to a holomorphic map $\widetilde{f}: M \rightarrow \mathbb{C P}$, which we call a holomorphic resolution of $f$. (See for example $[\mathrm{G}-\mathrm{H}]$.) We now recall known facts about $M$. Let $l_{o}$ be the line at infinity in $\mathbb{C P}^{2}\left(l_{o}:=\{(x: y: z): z=0\}\right)$. Then $M$ is obtained from $\mathbb{C P}^{2}$ by blowing up starting at a finite number of points on $l_{o}$. It is convenient to describe the blow up procedure by the following undirected graph $(W, E)$, where $W$ is the set of vertices and $E$ is the set of undirected edges. $l_{o}$ and all other $\mathbb{C P}^{1}$ obtained by the blow up are viewed as the vertices of $W$. For $v \in W$ we let $l_{v}$ denote the corresponding $\mathbb{C P}^{1}(o \in W)$. The undirected edge $(u, v)$ is in $E$ iff $l_{u} \cap l_{v} \neq \emptyset$. Then $(W, E)$ is a tree. We let $o$ be its root. Then there exists a unique orientation $E^{\prime} \subset W \times W$ of the undirected edges $E$ such that there exists a unique dipath from $o$ to any vertex $v \in W$. The terminal vertices $W$ of the directed tree $\left(W, E^{\prime}\right)$ are called the leaves of the tree $(W, E)$. Note that $L:=M \backslash \mathbb{C}^{2}=\bigcup_{v \in W} l_{v}$. View $\mathbb{C P}^{1}$ as $\mathbb{C} \cup\{\infty\}$. Set $L_{\infty}=\widetilde{f}^{-1}(\infty)$. Then there exists a subset $W(\infty) \subset W$ so that $L_{\infty}=\bigcup_{v \in W(\infty)} l_{v}$. Clearly, $o \in W(\infty)$.

(1.4) Theorem. Let $f: \mathbb{C}^{2} \rightarrow \mathbb{C}$ be a nonconstant polynomial map. Let $\widetilde{f}: M \rightarrow \mathbb{C P}^{1}$ be a holomorphic resolution of $f$. Then $L_{\infty}=\widetilde{f}^{-1}(\infty)$ is a connected divisor which induces a subtree $(W(\infty), E(\infty)) \subset(W, E)$ rooted at $o$. Let $v_{1}$ be a leaf of $(W(\infty), E(\infty))$ which is not a leaf of $(W, E)$. Then there is exactly one connected component $\left(W_{1}, E_{1}\right)$ of the forest $(\widehat{W}, \widehat{E})$ (induced by $\widehat{W}=W \backslash W(\infty)$ ) which is connected to $v_{1}$ at its root $o_{1} \in W_{1}$. Furthermore,

$$
\widetilde{f}\left(l_{o_{1}}\right)=\mathbb{C P}^{1}, \quad \widetilde{f}\left(l_{v}\right)=\text { const } \in \mathbb{C}, \quad v \in W_{1} \backslash o_{1} .
$$

See $[$ Ore $]$ and $[\mathrm{L}-\mathrm{W}]$.

(1.5) Corollary. Let the assumptions of Theorem (1.4) hold. Then there exits a minimal holomorphic resolution $\widetilde{f}: M \rightarrow \mathbb{C P}^{1}$ with the following property: Each connected component of the forest $(\widehat{W}, \widehat{E})$ consists of one vertex. That is, $M \backslash\left(\mathbb{C}^{2} \cup L_{\infty}\right)$ is the union $\bigcup_{v \in K} \widetilde{l}_{v}$ of $k:=|K|$ nonintersecting affine lines, $K:=W \backslash W(\infty)$, such that each $\tilde{f}: \widetilde{l}_{v} \rightarrow \mathbb{C}$ is a nonconstant polynomial map $\phi_{v}: \mathbb{C} \rightarrow \mathbb{C}$. Moreover

$$
\sum_{v \in K} \operatorname{deg} \phi_{v} \leq \operatorname{deg} f
$$

Proof. Let $M_{0}$ be any surface satisfying the assumption of Theorem (1.4). We blow $\mathbb{C P}^{2}$ along the rooted subtree $(W(\infty), E(\infty))$ to obtain the surface $M(\infty)$. Let $f_{0}: M(\infty) \rightarrow \mathbb{C P}^{1}$ be the rational map induced by $f$. Let $v_{1}$ be a leaf of the subtree $(W(\infty), E(\infty))$ as described in Theorem (1.4). Blow up at the corresponding point of $l_{v_{1}}$ to obtain $l_{o_{1}}$. Let $M_{1}$ be 
the resulting surface and $f_{1}: M_{1} \rightarrow \mathbb{C P}^{1}$ be the lifting of $f_{0}$. We claim that $f_{1}$ is holomorphic in some neighborhood of $l_{o_{1}}$. According to Theorem (1.4), $f_{1}$ may not be holomorphic at a finite number of points of $l_{o_{1}}$ at which we need to blow up to obtain $M_{0}$. However, in the neighborhood of these points, $\widetilde{f}$ and hence $f_{1}$ are bounded. By the Riemann extension theorem $f_{1}$ is holomorphic at these points. Hence $f_{1}$ is holomorphic in some neighborhood of $l_{o_{1}}$ in $M_{1}$. According to Theorem (1.4), $f_{1}: l_{o_{1}} \rightarrow \mathbb{C P}^{1}$ is a nontrivial rational map. Furthermore, $l_{o_{1}} \cap f_{1}^{-1}(\infty)=\left\{p_{1}\right\}$. Let $\widetilde{l}_{o_{1}}:=l_{o_{1}} \backslash\left\{p_{1}\right\} \sim \mathbb{C}$. Then $f_{1} \mid \widetilde{l}_{o_{1}}$ is given by a nonconstant polynomial map. Use the above arguments for all leaves of $(W(\infty), E(\infty))$ (which are not the leaves of $(V, E)$ ) to obtain the corollary for all $v \in K$. Recall that the closure of the affine curve $f=t$ in $\mathbb{C P}^{2}$ intersects the line at infinity in $\operatorname{deg} f$ points (counting with multiplicities). Clearly the closure of a generic $f=t$ in $M$ has $\sum_{v \in K} \operatorname{deg} \phi_{v}$ points. Hence $\sum_{v \in K} \operatorname{deg} \phi_{v} \leq \operatorname{deg} f$.

Let $f \in \mathbb{C}\left[\mathbb{C}^{2}\right]$ be a nonconstant polynomial. Let $\tilde{f}: M \rightarrow \mathbb{C P}^{1}$ be the minimal holomorphic resolution of $f$ as described in Corollary (1.5). Let

$$
V_{t}:=\left\{(x, y) \in \mathbb{C}^{2}: f(x, y)=t\right\}, \quad \Sigma_{t}=\widetilde{f}^{-1}(t), \quad t \in \mathbb{C} .
$$

Clearly,

$$
V_{t} \subset \Sigma_{t}, \quad \Sigma_{t}=V_{t} \cup \bigcup_{v \in K}\left(\Sigma_{t} \cap \widetilde{l}_{v}\right) .
$$

Let $\operatorname{CVV}(\widetilde{f}) \subset \mathbb{C}$ be the finite set such that, for each $t \in \operatorname{CVV}(\widetilde{f}), \Sigma_{t}$ contains at least one critical (singular) point of $\widetilde{f}$. Then any two surfaces $\Sigma_{t_{1}}, \Sigma_{t_{2}}, t_{1}, t_{2} \in \mathbb{C} \backslash \operatorname{CVV}(\widetilde{f})$, are homeomorphic. We call a fiber $\Sigma_{t}, t \in$ $\mathbb{C} \backslash \operatorname{CVV}(\tilde{f})$, a regular (compact) fiber. $f$ is called primitive if a regular fiber is connected, i.e. each regular fiber is homeomorphic to a fixed compact (orientable) Riemann surface $\Sigma$ of genus gen. If $f$ is not primitive, then there exists $h \in \mathbb{C}[\mathbb{C}]$ and a primitive polynomial $q \in \mathbb{C}\left[\mathbb{C}^{2}\right]$ so that $f=h(q)$ (see $[\mathrm{Suz}]$ ). In what follows we assume that $f$ is primitive unless otherwise stated.

For $t \in \operatorname{CVV}(\widetilde{f})$ the desingularized $\Sigma_{t}$ is either a compact Riemann surface whose genus is different from gen (hence smaller than gen), or a finite union of compact Riemann surfaces $(f-t$ is a reducible polynomial). We call such a fiber $\Sigma_{t}$ a singular (compact) fiber. Let $T$ be the union of all critical values of the polynomials $\phi_{v}, v \in K$, given in Corollary (1.5). That is, for each $t \in \mathbb{C} \backslash T, \Sigma_{t}$ intersects $\widetilde{l}_{v}$ in a fixed number $k(v)$ of points:

$$
\Sigma_{t} \cap \widetilde{l}_{v}=\left\{\zeta_{v, 1}(t), \ldots, \zeta_{v, k(v)}(t)\right\}, \quad t \in \mathbb{C} \backslash T .
$$

Furthermore, for each $t \in T$ there exists $v \in K$ so that $\Sigma_{t} \cap \widetilde{l}_{v}$ has less than $k(v)$ points. Let $\operatorname{CVV}(f)=\operatorname{CVV}(\widetilde{f}) \cup T$. Then each $V_{t}$ with $t \notin \operatorname{CVV}(f)$ 
is homeomorphic to $\Sigma$ punctured at $\kappa:=\sum_{v \in K} k(v)$ points. We call such a fiber $V_{t}$ a regular (affine) fiber. Furthermore, for each $t \in \operatorname{CVV}(f)$ the singular (affine) fiber $V_{t}$ is not homeomorphic to a regular fiber.

We view $\mathbb{C} \times \mathbb{C}$ (isomorphic to $\mathbb{C}^{2}$ ) as a subset of $\mathbb{C P}^{1} \times \mathbb{C P}^{1}$. Let

$$
L_{\infty}^{\prime}:=\mathbb{C P}^{1} \times \mathbb{C P}^{1} \backslash \mathbb{C} \times \mathbb{C}=\infty \times \mathbb{C P}^{1} \cup \mathbb{C P}^{1} \times \infty .
$$

Let $g \in \mathbb{C}\left[\mathbb{C}^{2}\right]$ and assume that $f, g$ are algebraically independent. Then the map $F=(f, g): \mathbb{C}^{2} \rightarrow \mathbb{C} \times \mathbb{C}$ is dominating. Let $\check{F}=(\check{f}, \check{g}): M \rightarrow \mathbb{C P}^{1} \times \mathbb{C P}^{1}$ be a holomorphic resolution of $F$ obtained as follows. We first blow up $\mathbb{C P}^{2}$ to obtain a minimal resolution $\widetilde{f}: M_{1} \rightarrow \mathbb{C P}^{1}$ as described in Corollary (1.5). Let $\widetilde{F}=(\widetilde{f}, \widetilde{g}): M_{1} \rightarrow \mathbb{C P}^{1} \times \mathbb{C P}^{1}$ be the lifting of $F$ to $M_{1}$. Then continue to blow up $M_{1}$ until we resolve the singularities of $\widetilde{g}$ to obtain $M$ and $\check{F}$. Let $(W, E)$ be the rooted tree corresponding to the blow ups performed to obtain $M$. Let $L_{\infty}=\check{F}^{-1}\left(L_{\infty}^{\prime}\right)$ and denote by $(W(\infty), E(\infty))$ the rooted subtree corresponding to $L_{\infty}$. Theorem (1.4) and Corollary (1.5) yield:

(1.6) Theorem. Let $F=(f, g): \mathbb{C}^{2} \rightarrow \mathbb{C} \times \mathbb{C}$ be a polynomial dominating map. Then there exists a minimal resolution $\check{F}=(\check{f}, \check{g}): M \rightarrow \mathbb{C P}^{1} \times$ $\mathbb{C P}^{1}$ with the following properties: $L_{\infty}=\check{F}^{-1}\left(L_{\infty}^{\prime}\right)$ is a connected divisor on $M$. Let $(\widehat{W}, \widehat{E})$ be the forest induced by $\widehat{W}:=W \backslash W(\infty)$. Then each connected component of the forest $(\widehat{W}, \widehat{E})$ consists of one vertex. That is, $M \backslash\left(\mathbb{C}^{2} \cup L_{\infty}\right)$ is the union $\bigcup_{v \in K} \widetilde{l}_{v}$ of $k:=|K|$ nonintersecting affine lines, $K:=W \backslash W(\infty)$, such that each $\check{f}, \check{g}: \widetilde{l}_{v} \rightarrow \mathbb{C}$ is a polynomial map $\phi_{v}, \psi_{v}: \mathbb{C} \rightarrow \mathbb{C}$ and at least one of them is nonconstant (for each $v \in K$ ).

(1.7) Proposition. Let the assumptions of Theorem (1.6) hold. Let $X:=$ $\check{F}^{-1}(\mathbb{C} \times \mathbb{C})=M \backslash L_{\infty}$. Then $X$ is a Stein manifold iff $F$ is locally proper, i.e. $F^{-1}(x, y)$ is a finite (possibly empty) set for each $(x, y) \in \mathbb{C} \times \mathbb{C}$.

Proof. Assume that $F$ is not locally proper. Then there exists $(a, b) \in$ $\mathbb{C} \times \mathbb{C}$ so that $V_{a} \supset F^{-1}(a, b) \supset U_{a}$, where $U_{a}$ is an irreducible component of $V_{a}$. Let $\bar{U}_{a} \subset M$ be the closure of $U_{a}$. Then $\check{g}\left(\bar{U}_{a}\right)=b$. Hence $\bar{U}_{a} \subset$ $\check{F}^{-1}(a, b) \subset X$ and $X$ is not a Stein manifold.

Conversely, assume that $X$ is not a Stein manifold. As $\check{F}: X \rightarrow \mathbb{C} \times \mathbb{C}$ is proper it follows that $X$ is holomorphically convex. Hence $X$ contains a compact connected one-dimensional complex space $U$. Since $M$ is a projective variety, $U$ must be a projective curve. Since $\widetilde{l}_{v}, v \in K$, is Stein it follows that $U \cap \widetilde{l}_{v}$ is a finite set, i.e. $U \cap \mathbb{C}^{2}$ is an infinite set. Clearly, $\check{F}(U) \subset \mathbb{C} \times \mathbb{C}$ must be a compact complex space. Since $\mathbb{C} \times \mathbb{C}$ is Stein it follows that $\check{F}(U)=(a, b)$ and $F^{-1}(a, b)$ is an infinite set. (See [G-R, p. 33].)

2. Monodromy. Let $f \in \mathbb{C}\left[\mathbb{C}^{2}\right]$ be a nonconstant primitive polynomial. Let $\tilde{f}: M \rightarrow \mathbb{C P}^{1}$ be the minimal resolution of $f$ as given in Corollary (1.4). 
Define $X:=\widetilde{f}^{-1}(\mathbb{C})=M \backslash L_{\infty}$. Corollary (1.5) yields that $X$ is a twodimensional simply connected manifold. ( $M$ is holomorphically convex but not Stein.) Let

$$
\begin{aligned}
& \operatorname{CPV}(f):=\bigcup_{t \in \operatorname{CVV}(f)} V_{t}, \quad \operatorname{CPV}_{1}(f):=\bigcup_{t \in \operatorname{CVV}(f)} \Sigma_{t}, \\
& B:=\mathbb{C} \backslash \operatorname{CVV}(f), \quad Y:=X \backslash \mathrm{CPV}_{1}(f), \quad Z:=\mathbb{C}^{2} \backslash \operatorname{CPV}(f) .
\end{aligned}
$$

Then $Y, Z$ are fiber bundles on $B$ given by the maps

$$
\tilde{f}: Y \rightarrow B, \quad f: Z \rightarrow B
$$

with fibers $\Sigma_{t}, V_{t}, t \in B$. Consider the $\mathbb{Z}$-bundles $A_{i} \rightarrow B, i=0,1$, with fibers $H_{1,0}(t):=H_{1}\left(\Sigma_{t}, \mathbb{Z}\right)$ for $i=0$ and $H_{1,1}(t):=H_{1}\left(V_{t}, \mathbb{Z}\right)$ for $i=1$ respectively for $t \in B$. Then $A_{i}$ is endowed with the unique locally trivial (Hurewicz) connection, which associates with any continuous path $\gamma:[0,1] \rightarrow B$ a $\mathbb{Z}$-isomorphism $D_{i}(\gamma): H_{1, i}(\gamma(0)) \rightarrow H_{1, i}(\gamma(1))$, $i=0,1$. Assume that $\gamma^{\prime}:[0,1] \rightarrow B$ is another continuous path with $\gamma(0)=\gamma^{\prime}(0)=t_{0}$ and $\gamma(1)=\gamma^{\prime}(1)$. If the closed curve $\gamma^{\prime-1} \gamma$ represents a trivial element in $\pi_{1}\left(B, t_{0}\right)$ then $D_{i}(\gamma)=D_{i}\left(\gamma^{\prime}\right), i=0,1$. The fundamental group $\pi_{1}\left(B, t_{0}\right)$ has the monodromy representations

$$
\phi_{0}^{\prime}: \pi_{1}\left(B, t_{0}\right) \rightarrow \operatorname{Aut}\left(H_{1}\left(\Sigma_{t_{0}}, \mathbb{Z}\right)\right), \quad \phi_{1}^{\prime}: \pi_{1}\left(B, t_{0}\right) \rightarrow \operatorname{Aut}\left(H_{1}\left(V_{t_{0}}, \mathbb{Z}\right)\right) .
$$

Let $\widetilde{l}_{v}, v \in K$, be an affine line on $M$ and consider the nonconstant polynomial map $\phi_{v}$ of degree $k(v)$ given in Corollary (1.5). Then

$$
\begin{array}{ll}
\kappa:=\sum_{v \in K} k(v), & \\
\phi_{v}^{-1}(t)=\left\{\zeta_{v, 1}(t), \ldots, \zeta_{v, k(v)}(t)\right\}, & v \in K, \\
\left\{\zeta_{1}(t), \ldots, \zeta_{\kappa}(t)\right\}=\bigcup_{v \in K} \phi_{v}^{-1}(t), & t \in B .
\end{array}
$$

Let $V_{t}$ be a regular fiber. Choose a basis in $H_{1}\left(V_{t}, \mathbb{Z}\right)$ as described at the beginning of $\S 1$. More precisely, $\gamma_{1}(t), \ldots, \gamma_{2}$ gen $(t) \subset V_{t}$ is a basis of the regular fiber $\Sigma_{t}$ of genus gen. For each $v \in K$ we have the simple Jordan curve $\gamma_{v, i}(t) \subset V_{t}$ whose interior in $\Sigma_{t}$ includes only $\zeta_{v, i}(t)$ for $i=1, \ldots, k(v)$. Then a basis of $H_{1}\left(V_{t}, \mathbb{Z}\right)$ consists of $\gamma_{j}(t), j=1, \ldots, 2$ gen, and the set $\left(\bigcup_{v \in K} \bigcup_{1 \leq i \leq k(v)}\left\{\gamma_{v, i}(t)\right\}\right) \backslash\left\{\gamma_{v^{\prime}, i^{\prime}}(t)\right\}$, for any curve $\gamma_{v^{\prime}, i^{\prime}}(t)$.

Choose a base point $t_{0} \in B$. Let $\pi_{1}=\pi_{1}\left(B, t_{0}\right)$ be the fundamental group of the base space. It is a free group on $l$ generators, where $\operatorname{CVV}(f)=$ $\left\{c_{1}, \ldots, c_{l}\right\}$. Consider the following set of generators $\tau_{1}, \ldots, \tau_{l}$ of $\pi_{1}\left(B, t_{0}\right)$. Each $\tau_{j}$ is a closed Jordan curve passing through $t_{0}$ so that its interior contains only the point $c_{j} \in \operatorname{CVV}(f)$ while all other points of $\operatorname{CVV}(f)$ are outside $\tau_{j}$. Furthermore, any pair $\tau_{i}, \tau_{j}$ intersects only at $t_{0}$. Each $\tau_{j}$ induces a monodromy diffeomorphism $\phi_{j}: \Sigma_{t_{0}} \rightarrow \Sigma_{t_{0}}$ which fixes the set $\Sigma_{t_{0}} \backslash V_{t_{0}}$, 
i.e. $\phi_{j}: V_{t_{0}} \rightarrow V_{t_{0}}$. Since $\pi_{1}\left(B, t_{0}\right)$ is a free group generated by $\left[\tau_{1}\right], \ldots,\left[\tau_{l}\right]$ we obtain the following homomorphisms:

$$
\begin{aligned}
& \phi_{0}: \pi_{1}\left(B, t_{0}\right) \rightarrow \operatorname{Diffeo}\left(\Sigma_{t_{0}}\right), \\
& \phi_{1}: \pi_{1}\left(B, t_{0}\right) \rightarrow \operatorname{Diffeo}\left(V_{t_{0}}\right), \\
& \phi_{2}: \pi_{1}\left(B, t_{0}\right) \rightarrow \operatorname{Perm}\left(\left\{\zeta_{1}\left(t_{0}\right), \ldots, \zeta_{\kappa}\left(t_{0}\right)\right\}\right) .
\end{aligned}
$$

Here, $\operatorname{Perm}(S)$ is the group of permutations on a finite set $S$. (The above homomorphisms are determined uniquely modulo the group of diffeomorphisms isotopic to the identity.)

Then $\phi_{0}, \phi_{1}$ induce the monodromy representations $\phi_{0}^{\prime}, \phi_{1}^{\prime}$ discussed above. Moreover, we have the following induced homomorphism:

$$
\phi_{2}^{\prime}: \pi_{1}\left(B, t_{0}\right) \rightarrow U_{t_{0}}, \quad U_{t_{0}}=\operatorname{span}\left(\gamma_{2 \text { gen }+1}\left(t_{0}\right), \ldots, \gamma_{2 \text { gen }+\kappa}\left(t_{0}\right)\right) .
$$

Note that the dimension of $U_{t_{0}}$ is $\kappa-1$. Furthermore, $\phi_{1}(\tau), \tau \in \pi_{1}\left(B, t_{0}\right)$, acts as a transitive subgroup of permutations on the curves $\gamma_{v, i}\left(t_{0}\right), i=$ $1, \ldots, k(v)$, for each $v \in K$. Let $\tau_{0} \in B$ be a circle of a big radius passing through $t_{0}$ so that $\mathrm{CVV}(f)$ lies inside this circle. Then $\phi_{0}^{\prime}\left(\tau_{i}\right), i=0, \ldots, l$, are called the local monodromies. (Each $\tau_{i}, i=1, \ldots, l$, encloses a finite singular fiber, and $\tau_{0}$ encloses the singular fiber at $\infty$.) Then all the eigenvalues of $\phi_{0}^{\prime}\left(\tau_{i}\right), i=0, \ldots, l$, are roots of unity and the Jordan blocks are of dimension 2 at most (see e.g. [Gri]). Clearly, $\phi_{2}^{\prime}(\tau), \tau \in \pi_{1}\left(B, t_{0}\right)$, is a semisimple matrix whose eigenvalues are roots of unity. Observe next that $\phi_{1}^{\prime}(\tau)$ can be viewed as a $2 \times 2$ lower block triangular matrix:

$$
\begin{aligned}
& \phi_{1}^{\prime}(\tau)=\left(a_{i j}(\tau)\right)_{i, j=1}^{N}=\left(A_{i j}(\tau)\right)_{i, j=1}^{2}, \quad A_{12}=0, \\
& \phi_{1}^{\prime}(\tau)\left[\gamma_{i}\left(t_{0}\right)\right]=\sum_{j=1}^{N} a_{j i}(\tau)\left[\gamma_{j}\left(t_{0}\right)\right], \quad i=1, \ldots, N .
\end{aligned}
$$

Here $A_{11}(\tau)$ and $A_{22}(\tau)$ represent the actions of $\tau$ on $H_{1}\left(\Sigma_{t_{0}}, \mathbb{Z}\right)$ and $U_{t_{0}}$ respectively. Hence all the eigenvalues of $\phi_{1}^{\prime}\left(\tau_{i}\right), i=0, \ldots, l$, are roots of unity.

Consider the complex vector bundles $E_{i} \rightarrow B, i=0$, 1, with fibers $\mathcal{H}^{1}\left(\Sigma_{t}\right)$ and $\mathcal{H}^{1}\left(V_{t}\right), t \in B$, respectively. $\left(\mathcal{H}^{1}\left(\Sigma_{t}\right)\right.$ denotes the subspace of holomorphic 1-forms on $\Sigma_{t}$.) The Gauss-Manin connection on $E_{1}$ is a holomorphic flat connection which is compatible with the Hurewicz connection (by (1.3)). The Gauss-Manin connection on $E_{0}$ is slightly more complicated. Observe first that $\operatorname{dim} \mathcal{H}^{1}\left(\Sigma_{t}\right)=$ gen. Then the Gauss-Manin connection is compatible with the Hurewicz connection by (1.1).

The Gauss-Manin connection induces the dual (cohomology) monodromies

$$
\phi_{1}^{*}: \pi_{1} \rightarrow \operatorname{Aut}\left(\mathcal{H}^{1}\left(V_{t_{0}}\right)\right), \quad \phi_{0}^{*}: \pi_{1} \rightarrow \operatorname{Aut}\left(\mathcal{H}^{1}\left(\Sigma_{t_{0}}\right)\right)
$$


A fundamental result due to Deligne [Del2] states that the representations $\phi_{0}^{*}$ and $\phi_{0}^{\prime}$ are semisimple.

(2.3) Proposition. Let $f \in \mathbb{C}\left[\mathbb{C}^{2}\right]$ be a nonconstant primitive polynomial. Then the eigenvalues of each $\phi_{0}^{\prime}(\tau), \tau \in \pi_{1}\left(B, t_{0}\right)$, are roots of unity iff $\phi_{0}\left(\pi_{1}\left(B, t_{0}\right)\right)$ is a finite group.

To prove this proposition we quote a theorem due to T. Laffey and the author:

(2.4) Theorem. Let $G$ be a subgroup of $\operatorname{GL}(n, \mathbb{Z})$ and assume that the eigenvalues of each element of $G$ are roots of unity. Then $G$ is virtually unipotent. That is, there exists a subgroup $G_{0}$ of $G$ of a finite index and $T \in \operatorname{GL}(n, \mathbb{Z})$ so that $T G_{0} T^{-1}$ is a subgroup of integer upper triangular matrices with 1 on the main diagonal.

Proof. Let $A \in G$. Then $\operatorname{det}(\lambda I-A)$ is a monic polynomial with integer coefficients. As all eigenvalues of $A$ are roots of unity it follows that $\operatorname{det}(\lambda I-A)$ is a product of cyclotomic polynomials $p_{1}(\lambda), \ldots, p_{m}(\lambda)$ where $\operatorname{deg} p_{i} \leq n, i=1, \ldots, m$. Hence the set of all possible polynomials $\operatorname{det}(\lambda I-A), A \in G$, is a finite set. Let $H$ be the Zariski closure of $G$ in $\operatorname{GL}(n, \mathbb{R})$ and denote by $H_{0}$ the identity component. Note that $H_{0}$ has a finite index $i$ in $H$. Clearly, $\operatorname{det}(\lambda I-B)=(\lambda-1)^{n}, B \in H_{0}$. Then there exists $T_{0} \in \mathrm{GL}(n, \mathbb{R})$ so that $T_{0} H_{0} T_{0}^{-1}$ is a subgroup of upper triangular matrices with 1 on the main diagonal [Kol, Prop. 40, p. 369]. Hence $G_{0}:=G \cap H_{0}$ has index $i$ in $G$. Since $G_{0} \in \operatorname{GL}(n, \mathbb{Z})$ it is straightforward to show that $T_{0}$ can be replaced by $T \in \mathrm{GL}(n, \mathbb{Z})$.

Proof of Proposition (2.3). Let $G:=\operatorname{Aut}\left(H_{1}\left(\Sigma_{t_{0}}, \mathbb{Z}\right)\right) \subset \operatorname{Sp}($ gen, $\mathbb{Z})$. Suppose first that $G$ is finite. Then the eigenvalues of each $A \in G$ are roots of unity. Assume now that the eigenvalues of each $A \in G$ are roots of unity. From Theorem (2.4) we deduce the existence of unipotent $G_{0} \leq G$ of a finite index. By Deligne's theorem $G$ is semisimple. Hence $G_{0}$ is semisimple and unipotent, which implies that it is trivial. Therefore $G$ is finite.

Let

$$
\begin{gathered}
\operatorname{Fix}\left(\phi_{1}^{\prime}\right):=\left\{a \in H_{1}\left(V_{t_{0}}, \mathbb{Z}\right): \phi_{1}(\tau)(a)=a, \tau \in \pi_{1}\left(B, t_{0}\right)\right\}, \\
\operatorname{Fix}\left(\phi_{1}^{*}\right):=\left\{\omega \in \mathcal{H}^{1}\left(V_{t_{0}}\right): \phi_{1}^{*}(\tau)(\omega)=\omega, \tau \in \pi_{1}\left(B, t_{0}\right)\right\}, \\
\operatorname{fix}_{1}(f):=\operatorname{dim} \operatorname{Fix}\left(\phi_{1}^{\prime}\right), \quad \operatorname{fix}^{1}(f):=\operatorname{dim} \operatorname{Fix}\left(\phi_{1}^{*}\right) .
\end{gathered}
$$

(2.5) Theorem. Let $f \in \mathbb{C}\left[\mathbb{C}^{2}\right]$ be a nonconstant primitive polynomial. Then the monodromy action on the regular affine fiber $V_{t}$ has a nontrivial fixed element in $\mathcal{H}^{1}\left(V_{t}\right)$ iff the polynomial $f-c$ is reducible for some $c \in \mathbb{C}$.

Proof. Suppose first that $f-c=f_{1} f_{2}$, where $f_{1}, f_{2}$ are nonconstant polynomials. Then $\omega:=d f_{1} /\left(2 \pi \sqrt{-1} f_{1}\right)$ is a nonzero 1 -form on $Z$. The 
restriction $\omega_{t}$ to a regular fiber $V_{t}$ gives an element $\omega_{t} \in H^{1}\left(V_{t}, \mathbb{Z}\right) .\left(\int_{\gamma} \omega_{t}\right.$ is the change of the argument of $f_{1}$ along $\gamma \subset V_{t}$.) Hence the monodromy acts on $\omega_{t}$. As $f_{1}$ cannot be a constant function on $V_{t}$ it follows that $\omega_{t}$ is a nontrivial element of $\mathcal{H}^{1}\left(V_{t}\right)$ which is fixed under the monodromy action.

Assume now that each fiber $V_{t}, t \in \mathbb{C}$, is irreducible. According to Libgober [Lib1], $H_{1}(Z, \mathbb{Z})$ is a free abelian group of rank $l$. Thus any element $\varrho \in H^{1}(Z, \mathbb{Z})$ is of the form

$$
\varrho=\frac{1}{2 \pi \sqrt{-1}} \sum_{i=1}^{l} \frac{m_{i} d f_{i}}{f_{i}}, \quad m_{i} \in \mathbb{Z}, i=1, \ldots, l .
$$

Let $B_{0} \subset B$ be the union of $l$ closed Jordan curves $\tau_{1}, \ldots, \tau_{l}$ which were described at the beginning of this section. ( $B_{0}$ is a bouquet of $l$ circles.) Hence, $\pi_{1}\left(B, t_{0}\right)=\pi_{1}\left(B_{0}, t_{0}\right)$. Moreover, $B_{0}$ is a deformation retract of $B$. Let $Z_{0} \subset Z$ be the fiber bundle $Z_{0}=f^{-1}\left(B_{0}\right)$. Then $Z_{0}$ is a deformation retract of $Z$. (Deform each point on $V_{t}$ along the gradient flow $\left(\bar{f}_{x}, \bar{f}_{y}\right)$. See [Mil] for details.) In particular, $H_{1}\left(Z_{0}, \mathbb{Z}\right)$ is a free abelian group on $l$ generators. (Note that there is a 2-complex $Z_{1}$ which is a deformation retract of $Z_{0}$ and which can be constructed explicitly from $Z_{0}$ according to [Lib2].) Then each element $\varrho \in H_{1}\left(Z_{0}, \mathbb{Z}\right)$ is still given by (2.6). Note that for each closed curve $\gamma \subset V_{t}, t \in B$, we have $\varrho(\gamma)=0$. Consider the injection $\iota: H_{1}\left(V_{t_{0}}, \mathbb{Z}\right) \rightarrow H_{1}\left(Z_{0}, \mathbb{Z}\right)$. The equality $\varrho(\gamma)=0$ yields that $\iota$ is trivial.

Assume to the contrary that we have a nontrivial fixed element $\omega \in$ $H^{1}\left(V_{t_{0}}\right)$. Since monodromy is generated by $l$ integer-valued matrices it follows that we can assume that $\omega \in H^{1}\left(V_{t_{0}}, \mathbb{Z}\right)$. In particular, any element $\beta$ in the kernel of $\omega$ is mapped to another element of the kernel under the action of monodromy. As $\iota$ is trivial it follows that for any $[\gamma] \in H_{1}\left(V_{t_{0}}, \mathbb{Z}\right)$ the subspace spanned by $\phi([\tau])([\gamma]),[\tau] \in \pi_{1}\left(B_{0}, t_{0}\right)$, is $H_{1}\left(V_{t_{0}}, \mathbb{Z}\right)$. This contradicts the existence of $\omega$.

A. Libgober pointed out that Theorem (2.5) can be generalized as follows:

(2.7) TheOREM. Let $f \in \mathbb{C}\left[\mathbb{C}^{2}\right]$ be a nonconstant primitive polynomial. Then fix $^{1}(f)=\delta(f)($ see $(0.1))$.

Proof. Assume that $V_{c}$ reduces to $n$ irreducible curves. That is, $f-c=$ $g_{1}^{m_{1}} \ldots g_{n}^{m_{n}}$, where $g_{1}, \ldots, g_{n}$ are nontrivial coprime factors of $f-c$. Then $\frac{d g_{1}}{g_{1}}\left|V_{t_{0}}, \ldots, \frac{d g_{n}}{g_{n}}\right| V_{t_{0}} \in \operatorname{Fix}\left(\phi_{1}^{*}\right)$. Note that these $n 1$-forms satisfy exactly one relation:

$$
\sum_{i=1}^{n} m_{i} \frac{d g_{i}}{g_{i}} \mid V_{t_{0}}=0
$$

Hence $\operatorname{fix}^{1}(f) \geq \delta(f)$. According to [Lib1], $\operatorname{dim} H^{1}(Z, \mathbb{C})=l+\delta(f)$. A basis of $H^{1}(Z, \mathbb{C})$ is given by $d f_{i} / f_{i}, i=1, \ldots, l+\delta(f)$, where $Z\left(f_{1}\right), \ldots, Z\left(f_{l+\delta(f)}\right)$ 
are the irreducible components of $\operatorname{CPV}(f)$. As observed above, only $\delta(f)$ of those give rise to linearly independent forms in $\operatorname{Fix}\left(\phi_{1}^{*}\right)$.

(2.8) Lemma. Let $f \in \mathbb{C}\left[\mathbb{C}^{2}\right]$ be a nonconstant primitive polynomial. Then fix $_{1}(f) \geq|K|-1 \geq m-1$, where $K$ is given in Corollary (1.5) and $m$ is the number of points at which the closure of any $V_{t}$ in $\mathbb{C P}^{2}$ intersects the line at infinity.

Pr o of. From the definition of $K$ it follows that $|K| \geq m$. Let $v_{1}, v_{2} \in K$, $v_{1} \neq v_{2}$. Then the element

$$
[\gamma(t)]:=k\left(v_{2}\right) \sum_{i=1}^{k\left(v_{1}\right)}\left[\gamma_{v_{1}, i}(t)\right]-k\left(v_{1}\right) \sum_{i=1}^{k\left(v_{2}\right)}\left[\gamma_{v_{2}, i}(t)\right]
$$

is a nonzero element which is fixed under the action of the monodromy. Hence $\operatorname{fix}_{1}(f) \geq|K|-1$.

We now present the following example of Deligne which shows that the monodromy action on an affine fiber $V_{t}$ does not have to be semisimple.

(2.9) Proposition. Let $f=x y^{2}+x^{2}+y$. Then each fiber $f=t$ is irreducible, and the regular fiber $V_{t}$ is an elliptic curve (of genus one) punctured at two points which are fixed under the monodromy action. The monodromy action on $H_{1}\left(V_{t}, \mathbb{C}\right)\left(\right.$ and on $\left.H^{1}\left(V_{t}, \mathbb{C}\right)\right)$ is not semisimple.

Proof. Consider the fiber $\Sigma_{t}$. Let $(x, y) \mapsto x$ be the projection $\psi: \Sigma_{t} \rightarrow$ $\mathbb{C P}^{1}$. Then $\Sigma_{t}$ is a double (branched) cover of $\mathbb{C P}^{1}$. Consider $f(x, y)=t$. Then

$$
y=\frac{-1 \pm \sqrt{1-4 x\left(x^{2}-t\right)}}{2 x} .
$$

The branching points over $\mathbb{C}$ are given by $4 x\left(x^{2}-t\right)=1$. For $t^{3} \neq 27 / 64$ we have exactly 3 branching points over $\mathbf{C}$, each one of multiplicity 2 . In particular, for all the above values $V_{t}$ is irreducible. For $t^{3}=27 / 64$ we have exactly one branching point of multiplicity 2 . Hence the three singular fibers are also irreducible. The point at infinity $(1: 0: 0)$ is also a branching point of multiplicity 1 . The point at infinity $(0: 1: 0)$ is a regular point. Use the Riemann-Hurwitz formula to deduce that the regular fibers $\Sigma_{t}$ are elliptic curves, while the three exceptional fibers are $\mathbb{C P}$. Since each $V_{t}$ is irreducible we deduce that

$$
H_{1}\left(\mathbb{C}^{2}-\bigcup_{t^{3}=27 / 64} V_{t}, \mathbb{Z}\right)=\mathbb{Z}^{3} .
$$

Theorem (2.5) yields that fix $^{1}(f)=0$. Lemma (2.8) implies that fix $1(f) \geq$ $2-1=1$. Since $\operatorname{fix}_{1}(f) \neq \operatorname{fix}^{1}(f)$ it follows that $\phi_{1}^{\prime}\left(\pi_{1}\left(B, t_{0}\right)\right)$ is not semisimple. 
(2.10) Corollary. Let $f \in \mathbb{C}\left[\mathbb{C}^{2}\right]$ be a primitive polynomial so that a regular fiber $V_{t}$ is a Riemann sphere $\left(\Sigma_{t}=\mathbb{C P}^{1}\right)$ punctured at two points at least. Suppose furthermore that the monodromy does not act transitively on $\Sigma_{t} \backslash V_{t}$ $(|K|>1)$. Then $f-c$ is reducible for some $c \in \mathbb{C}$.

Proof. The monodromy acts as a subgroup of permutations on $H_{1}\left(V_{t}, \mathbb{Z}\right)$. Hence the action of the monodromy is semisimple. Therefore $\mathrm{fix}^{1}(f)=\mathrm{fix}_{1}(f) \geq|K|-1>0$. Theorem (2.5) yields that $f-c$ is reducible for some $c$.

$f=x y$ is an example satisfying the conditions of Corollary (2.10).

(2.11) TheOREM. Let $f \in \mathbb{C}\left[\mathbb{C}^{2}\right]$ be a primitive polynomial so that a regular fiber $V_{t}$ is either a punctured Riemann sphere or a punctured elliptic curve (torus). Suppose that there exists $g \in \mathbb{C}\left[\mathbb{C}^{2}\right]$ and a finite set $T \subset \mathbb{C}$ such that $g: V_{t} \rightarrow \mathbb{C}$ is not injective and has no critical (ramification) points for $t \in \mathbb{C} \backslash T$. Then $f-c$ is reducible for some $c \in \mathbb{C}$.

Proof. Let $g_{t}: \Sigma_{t} \rightarrow \mathbb{C P}^{1}$ be the extension of $g: V_{t} \rightarrow \mathbb{C}$. Without loss of generality we may assume that $T \supset \operatorname{CVV}(f)$. Suppose first that $\Sigma_{t}=\mathbb{C P}^{1}$. Since $g: V_{t} \rightarrow \mathbb{C}$ is not an injection the degree of $g_{t}$ is two at least. Hence $g_{t}$ has to have at least two critical points. Moreover, at least one of the critical points is not in $g_{t}^{-1}(\infty)$. (Recall that an unramified cover of $\mathbb{C}$ is a homeomorphism.) Clearly, $g_{t}^{-1}(\infty)$ is invariant under the action of monodromy. According to Corollary (2.10), $f-c$ is reducible.

Assume now that the genus of a regular fiber $\Sigma_{t}$ is equal to 1 . Since $g_{t}$ has degree two at least and has no ramification points on $V_{t}$, the 1-form $d g_{t}$ has zeros and poles on $\Sigma_{t} \backslash V_{t}$. Let $\omega_{t}$ be the holomorphic 1-form on $\Sigma_{t}$. Recall that $\omega_{t}$ is unique up to a nonzero factor. Normalize $\omega_{t}$ by assuming that $\int_{\gamma_{1}(t)} \omega_{t}=1$. Then $\omega_{t}$ is locally continuous in the parameter $t$. (However, the monodromy will act on $\omega_{t}$.) Let $h_{t}:=\omega_{t} / d g_{t}: \Sigma_{t} \rightarrow \mathbb{C P}^{1}$. Then $h_{t}$ is a nonconstant function such that all poles and zeros of $h_{t}$ lie on $\Sigma_{t} \backslash V_{t}$. Consider the nontrivial 1-form $\theta_{t}:=d h_{t} /\left(2 \pi \sqrt{-1} h_{t}\right) \in \mathcal{H}^{1}\left(V_{t}\right)$. As in the proof of Theorem (2.5) we deduce that $\theta_{t} \in H^{1}\left(V_{t}, \mathbb{Z}\right)$ is a nontrivial invariant 1-form under the monodromy action. Theorem (2.5) implies that $f-c$ is reducible for some $c$.

The pair $f=x y, g=x^{2}$ satisfies the assumptions of Theorem (2.11). We do not know if Theorem (2.11) generalizes to a primitive $f$ such that the regular fiber $\Sigma_{t}$ has genus two at least. We prove a weaker version for the higher genus case:

(2.12) Proposition. Let $f \in \mathbb{C}\left[\mathbb{C}^{2}\right]$ be a primitive polynomial so that a regular fiber $V_{t}$ is a punctured Riemann surface of genus two at least. Suppose that there exists $g \in \mathbb{C}\left[\mathbb{C}^{2}\right]$ and a finite set $T \subset \mathbb{C}$ such that $g: V_{t} \rightarrow \mathbb{C}$ is 
not injective and has no critical (ramification) points for $t \in \mathbb{C} \backslash T$. Assume furthermore that $f_{x} d g / d y$ is not a constant function on $V_{t}$ for $t \in \mathbb{C} \backslash T$. Then $f-c$ is reducible for some $c \in \mathbb{C}$.

Proof. Observe that $d f=f_{x} d x+f_{y} d y$. Hence on a regular fiber $V_{t}$, $\omega_{t}:=d y / f_{x}=-d x / f_{y} \in \mathcal{H}^{1}\left(V_{t}\right)$. Let $h_{t}$ be defined as in the proof of Theorem (2.11). Our assumptions yield that $h_{t}$ is a nonconstant function whose poles and zeros lie on $\Sigma_{t} \backslash V_{t}$. Then the arguments of the proof of Theorem (2.11) yield the proposition.

3. 1-forms. Let $Y$ be a smooth projective variety and $X \subset Y$ be a quasi-projective variety. Denote by $\mathcal{O}_{\mathrm{r}}(X)$ and $\Omega_{\mathrm{r}}^{p}(X)$ all rational functions and rational $p$-forms respectively on $Y$ which are holomorphic on $X$. Denote by $\mathcal{H}^{p}(X), p \geq 1$, the space of all closed forms in $\Omega_{\mathrm{r}}^{p}(X)$ modulo $d \Omega_{\mathrm{r}}^{p-1}(X)$ $\left(\Omega_{\mathrm{r}}^{0}(X):=\mathcal{O}_{\mathrm{r}}(X)\right)$. Let $\omega \in \Omega_{\mathrm{r}}^{1}\left(\mathbb{C}^{2}\right)$. Then

$$
\omega=p(x, y) d x+q(x, y) d y,
$$

where $p, q \in \mathbb{C}\left[\mathbb{C}^{2}\right]$. Let $f \in \mathbb{C}\left[\mathbb{C}^{2}\right]$ and assume that $\widetilde{V}_{t}$ is a smooth irreducible component of $V_{t}$. Then $\omega_{t} \in \Omega_{\mathrm{r}}^{1}\left(\widetilde{V}_{t}\right)$ is the restriction of $\omega$ to the fiber $V_{t}$.

(3.2) Lemma. Let $f \in \mathbb{C}\left[\mathbb{C}^{2}\right]$ be a nonconstant primitive polynomial. Let $\widetilde{V}_{t}$ be a smooth irreducible component of the fiber $V_{t}$ and assume that $\alpha \in$ $\Omega_{\mathrm{r}}^{1}\left(\widetilde{V}_{t}\right)$. Then there exists a 1 -form $\omega \in \Omega_{\mathrm{r}}^{1}\left(\mathbb{C}^{2}\right)$ such that $\alpha=\omega_{t}$.

Proof. Assume that $\widetilde{V}_{t}=Z(u)$ for some irreducible $u$. As $\widetilde{V}_{t}$ is smooth it follows that

$$
\theta_{t}:=\frac{d y}{u_{x}}\left|\widetilde{V}_{t}=-\frac{d x}{u_{y}}\right| \widetilde{V}_{t} \in \Omega_{\mathrm{r}}^{1}\left(\widetilde{V}_{t}\right) .
$$

Furthermore, $\theta_{t}$ does not have zeros on $\widetilde{V}_{t}$. We first prove the lemma for $\theta_{t}$. Denote by $C$ all the critical values of the map $u: \mathbb{C}^{2} \rightarrow \mathbb{C}$. Let $h=\prod_{\tau \in C}(\tau-u)$. Hence $h$ vanishes at every critical point of $u$. The Hilbert Nullstellensatz yields

$$
h^{m}=-h_{1} u_{y}+h_{2} u_{x}, \quad h_{1}, h_{2} \in \mathbb{C}\left[\mathbb{C}^{2}\right],
$$

for some integer $m \geq 1$. Then $h^{m} \mid \widetilde{V}_{t}=a:=\prod_{\tau \in C} \tau^{m} \neq 0$. Let $\varrho=\frac{1}{a}\left(h_{1} d x+\right.$ $\left.h_{2} d y\right) \in \Omega_{\mathrm{r}}^{1}\left(\mathbb{C}^{2}\right)$. Hence $\varrho_{t}=\theta_{t}$. Let $\alpha \in \Omega_{\mathrm{r}}^{1}\left(\widetilde{V}_{t}\right)$. Then $\alpha / \theta_{t} \in \mathcal{O}_{\mathrm{r}}\left(\widetilde{V}_{t}\right)$. Since $\widetilde{V}_{t}$ is smooth, $\alpha / \theta_{t}=r \mid \widetilde{V}_{t}, r \in \mathbb{C}\left[\mathbb{C}^{2}\right]$. Then $\omega:=r \varrho$ satisfies the conditions of the lemma.

(3.3) Corollary. Let $f \in \mathbb{C}\left[\mathbb{C}^{2}\right]$ be a nonconstant primitive polynomial. Assume furthermore that $f$ does not have singular points. Then there exists $\omega \in \Omega_{\mathrm{r}}^{1}\left(\mathbb{C}^{2}\right)$ so that the restriction of $\omega$ to any irreducible component of $V_{t}$ is the 1 -form $\theta_{t}:=d y / f_{x}=-d x / f_{y} \in \mathcal{H}^{1}\left(V_{t}\right)$.

Proof. In the proof of Lemma (3.2) choose $h=1$. 
Let $f \in \mathbb{C}\left[\mathbb{C}^{2}\right]$ be a nonconstant primitive polynomial. Consider the complex vector bundle $E_{1} \rightarrow B$ with fiber $\mathcal{H}^{1}\left(V_{t}\right)$, as defined in $\S 2$. Clearly, any $\omega \in \Omega_{\mathrm{r}}^{1}\left(\mathbb{C}^{2}\right)$ induces a holomorphic section by viewing $\omega_{t} \in \Omega_{\mathrm{r}}^{1}\left(V_{t}\right)$ as an element in $\mathcal{H}^{1}\left(V_{t}\right)$. Usually, the set of all holomorphic sections obtained from the restriction of $\Omega_{\mathrm{r}}^{1}\left(\mathbb{C}^{2}\right)$ is too small. We now introduce the "right" classes of holomorphic and rational sections $\mathcal{O}_{\mathrm{r}}\left(E_{1}\right), \mathcal{R}\left(E_{1}\right)$ as follows. Let $\omega$ be a rational 1 -form on $\mathbb{C}^{2}$ of the form (3.1) where $p, q \in \mathbb{C}\left(\mathbb{C}^{2}\right)$. We say that $\omega$ defines a rational section on $E_{1}$ if there exists a finite set $T \subset \mathbb{C}$ so that $\omega_{t} \in \Omega_{\mathrm{r}}^{1}\left(V_{t}\right)$ for all $t \in \mathbb{C} \backslash(T \cup \mathrm{CVV}(f))$. Then the restriction of $\omega$ to all but a finite number of $V_{t}$ gives the element $\omega_{t} \in \mathcal{H}^{1}\left(V_{t}\right)$. We will abuse the notation by denoting this section by $\omega$ and its value on $\mathcal{H}^{1}\left(V_{t}\right)$ by $\omega_{t}$. (For a rational 1-form $\omega_{i}$ which defines a rational section in $E_{1}$ we denote by $\omega_{i, t}$ its restriction to $\mathcal{H}^{1}\left(V_{t}\right)$ when this restriction exists.) We denote the set of these sections by $\mathcal{R}\left(E_{1}\right)$. Denote by $\mathcal{O}_{\mathrm{r}}\left(E_{1}\right) \subset \mathcal{R}\left(E_{1}\right)$ all the rational sections which are holomorphic at each $t \in B$. Let $\mathcal{F} \subset \mathbb{C}\left(\mathbb{C}^{2}\right)$ be the field of all rational functions in the variable $f$ :

$$
\mathcal{F}:=\{a: a=b(f), b \in \mathbb{C}(\mathbb{C})\} .
$$

Clearly, if $\omega \in \mathcal{R}\left(E_{1}\right)$ then $a \omega \in \mathcal{R}\left(E_{1}\right)$ for any $a \in \mathcal{F}$. Hence $\mathcal{R}\left(E_{1}\right)$ is a vector space over $\mathcal{F}$. Two rational 1 -forms $\omega, \theta$ define the same rational section if there exists a finite set $T \subset \mathbb{C}$ such that $\omega_{t}$ and $\theta_{t}$ represent the same element in $\mathcal{H}^{1}\left(V_{t}\right)$ for all $t \in \mathbb{C} \backslash(T \cup \operatorname{CVV}(f))$. Let

$$
\mathcal{F}\left[\mathbb{C}^{2}\right]:=\left\{a \in \mathbb{C}\left(\mathbb{C}^{2}\right): a=b / c(f), b \in \mathbb{C}\left[\mathbb{C}^{2}\right], c \in \mathbb{C}[\mathbb{C}]\right\} .
$$

(3.4) Theorem. Let $f, g \in \mathbb{C}\left[\mathbb{C}^{2}\right]$ be such that $f$ is primitive and $F=(f, g)$ : $\mathbb{C}^{2} \rightarrow \mathbb{C}^{2}$ is a dominating map. Then any rational 1 -form

$$
\varrho:=r \frac{d g}{\operatorname{det} J(F)}, \quad r \in \mathcal{F}\left[\mathbb{C}^{2}\right],
$$

defines a rational section in $\mathcal{R}\left(E_{1}\right)$. Moreover, any rational section in $\mathcal{R}\left(E_{1}\right)$ is given by some @ of the above form. Furthermore, the dimension of $\mathcal{R}\left(E_{1}\right)$ as a vector space over $\mathcal{F}$ is $N:=\operatorname{dim} \mathcal{H}^{1}\left(V_{t}\right)=2$ gen $+k-1, t \in B$.

Proof. Recall that $\theta_{t}:=\frac{d y}{f_{x}}\left|V_{t}=-\frac{d x}{f_{y}}\right| V_{t} \in \Omega_{\mathrm{r}}^{1}\left(V_{t}\right), t \in B$, is a nonvanishing 1-form. Let $T \subset \mathbb{C}$ be a finite set such that $g_{t}:=g \mid V_{t}$ is a nonconstant map. A straightforward calculation shows that $\left.\frac{d g}{\operatorname{det} J(F)}\right|_{t}=\theta_{t}, t \in B \backslash T$. Hence any $\varrho$ of the form (3.5) induces a rational section in $E_{1}$. Suppose that $\omega$ is a rational 1-form on $\mathbb{C}^{2}$ of the form (3.1) such that $\omega_{t} \in \Omega_{\mathrm{r}}^{1}\left(V_{t}\right)$ for all but a finite number of $t$. Let $r=-p f_{x}+q f_{y}$ and assume that $\varrho$ is defined by (3.5). Then $\omega_{t}=\varrho_{t}$. Hence $r \mid V_{t} \in \mathcal{O}_{\mathrm{r}}\left(V_{t}\right)$ for all but a finite number of $t$, i.e. $r \in \mathcal{F}\left[\mathbb{C}^{2}\right]$.

We now show that the dimension of $\mathcal{R}\left(E_{1}\right)$ is $N$. For $t_{0} \in B$ fix a basis of $N$ elements in $H_{1}\left(V_{t_{0}}, \mathbb{Z}\right)$ and $N$-forms in $\Omega_{\mathrm{r}}^{1}\left(V_{t_{0}}\right)$ which satisfy (1.3) 
(as described in $\S 1$ ). Use Lemma (3.2) and the above arguments to deduce that there exist $p_{1}, \ldots, p_{N} \in \mathbb{C}\left[\mathbb{C}^{2}\right]$ such that the restrictions of the 1 -forms $\varrho_{j}:=p_{j} d g / \operatorname{det} J(F), j=1, \ldots, N$, to $V_{t_{0}}$ satisfy (1.3). Hence $\varrho_{j} \mid V_{t}, j=$ $1, \ldots, N$, form a basis for $\mathcal{H}^{1}\left(V_{t}\right)$ for all but a finite number of $t$. This shows that $\operatorname{dim}_{\mathcal{F}} \mathcal{R}\left(E_{1}\right) \geq N$. Let $\varrho$ be of the form (3.5). Then for all but a finite number of $t$ we have unique $a_{1}(t), \ldots, a_{n}(t)$ so that $\varrho-\sum_{i=1}^{N} a_{i}(t) \varrho_{i} \mid V_{t}$ is an exact 1-form on $V_{t}$. Hence $a_{1}(t), \ldots, a_{N}(t)$ are holomorphic functions on $\mathbb{C} \backslash T$ for a finite set $T \subset \mathbb{C}$.

We claim that each $a_{i}(t)$ is meromorphic at $T$ and at $\infty$. For $t_{1} \in$ $T \backslash \mathrm{CVV}(f)$ the argument is quite straightforward. Consider $N$ 1-forms $\widetilde{\varrho}_{1}, \ldots, \widetilde{\varrho}_{N} \in \Omega_{\mathrm{r}}^{1}\left(\mathbb{C}^{2}\right)$ whose restriction to $V_{t_{1}}$ gives a basis in $\mathcal{H}^{1}\left(V_{t_{1}}\right)$. Then $\widehat{\varrho}:=\varrho-\sum_{i=1}^{N} \widetilde{a}_{i}(t) \widetilde{\varrho}_{i} \mid V_{t}$ are exact 1-forms on $V_{t}$ where $\left|t-t_{1}\right|<\varepsilon$ for some $\varepsilon>0$. Here $\widetilde{a}_{1}(t), \ldots, \widetilde{a}_{N}(t)$ are holomorphic in $\left|t-t_{1}\right|<\varepsilon$. Change the basis from $\widetilde{\varrho}_{1}, \ldots, \widetilde{\varrho}_{N}$ to $\varrho_{1}, \ldots, \varrho_{N}\left(\right.$ in $\left.\mathcal{R}\left(E_{1}\right)\right)$ to deduce that $a_{1}(t), \ldots, a_{N}(t)$ are meromorphic at $t_{1}$. For $t_{1} \in \mathrm{CVV}(f) \cup \infty$ we have to use the growth estimates (see e.g. [Mal]) to deduce that $a_{1}(t), \ldots, a_{N}(t)$ are meromorphic at $t_{1}$. Hence $a_{1}(t), \ldots, a_{N}(t)$ are rational functions. Thus $\widehat{\varrho}_{t}$ is a trivial (0) section for all but a finite number of $t$. Therefore $\varrho_{1}, \ldots, \varrho_{N}$ form a basis of rational sections in $\mathcal{R}\left(E_{1}\right)$ over $\mathcal{F}$.

Let $\varrho_{1}, \ldots, \varrho_{N}$ be a basis of rational sections in $\mathcal{R}$. Then $\varrho_{1}, \ldots, \varrho_{N}$ is called a basis of $\mathcal{O}_{\mathrm{r}}\left(E_{1}\right)$ if $\varrho_{1}, \ldots, \varrho_{N} \in \mathcal{O}_{\mathrm{r}}\left(E_{1}\right)$ and for each $t \in B$, $\varrho_{1, t}, \ldots, \varrho_{N, t}$ is a basis for $\mathcal{H}^{1}\left(V_{t}\right)$.

(3.6) Lemma. Let the assumptions of Theorem (3.4) hold. Then $\mathcal{O}_{\mathrm{r}}\left(E_{1}\right)$ has a basis of holomorphic sections $\varrho_{1}, \ldots, \varrho_{N}$, given by the restrictions of the rational 1-forms

$$
\varrho_{j}=p_{j} \frac{d g}{\operatorname{det} J(F)}, \quad p_{j} \in \mathcal{F}\left[\mathbb{C}^{2}\right], j=1, \ldots, N .
$$

Let

$$
\widetilde{\varrho}_{i}=\sum_{j=1}^{N} a_{i j}(t) \varrho_{j}, \quad i=1, \ldots, N, \quad A(t):=\left(a_{i j}(t)\right)_{i, j=1}^{N} \text {. }
$$

Then $\widetilde{\varrho}_{1}, \ldots, \widetilde{\varrho}_{N}$ is a holomorphic basis in $\mathcal{O}_{\mathrm{r}}\left(E_{1}\right)$ iff $a_{i j}(t), i, j=1, \ldots, N$, are rational functions which are holomorphic on $B$ and $\operatorname{det} A(t)$ does not vanish on $B$.

P r o of. Use Lemma (3.2) to deduce the existence of $\omega_{1}, \ldots, \omega_{N} \in \Omega_{\mathrm{r}}^{1}\left(\mathbb{C}^{2}\right)$ so that $\omega_{1, t_{0}}, \ldots, \omega_{N, t_{0}}$ form a basis in $\mathcal{H}^{1}\left(V_{t_{0}}\right), t_{0} \in B$. As pointed out in the proof of Theorem (3.4) we may assume that $g=y$, i.e. $d g / \operatorname{det} J(F)=d y / f_{x}$. Fix $t \in B$ and a basis $\left[\gamma_{i}(t)\right], i=1, \ldots, N$, in $H_{1}\left(V_{t}, \mathbb{Z}\right)$ as in $\S 1$. Let 


$$
b(t)=\left(\operatorname{det}\left(\int_{\gamma_{i}(t)} \omega_{j}\right)_{i, j=1}^{N}\right)^{2} .
$$

Note that $b(t)$ does not depend on the choice of the basis. Moreover, $b(t)$ is holomorphic on $B$. Because of the growth conditions on $\operatorname{CVV}(f) \cup\{\infty\}$ (see e.g. [Mal]), it follows that $b(t)$ is meromorphic on $\operatorname{CVV}(f) \cup\{\infty\}$. Hence $b(t)$ is a rational function which is holomorphic on $B$. Let $T \subset B$ be the zero set of $b$ in $B$. If $T=\emptyset$ then $\omega_{i}, i=1, \ldots, N$, form a basis in $\mathcal{O}_{\mathrm{r}}\left(E_{1}\right)$ as claimed. Assume now that $b\left(t_{1}\right)=0$ for some $t_{1} \in B$. That is, $\omega_{i, t_{1}}, i=1, \ldots, N$, are linearly dependent elements of $\mathcal{H}^{1}\left(V_{t_{1}}\right)$. Let

$$
\widetilde{\omega}_{i}=\sum_{j=1}^{N} c_{i j} \omega_{j}, \quad i=1, \ldots, N,\left(c_{i j}\right)_{i, j=1}^{N} \in \operatorname{SL}(N, \mathbb{C}),
$$

be such that $\widetilde{\omega}_{1} \mid V_{t_{1}}$ is a zero element in $\mathcal{H}^{1}\left(V_{t_{1}}\right)$. Hence $\widehat{\omega}_{1}:=\widetilde{\omega}_{1} /\left(f-t_{1}\right)$ induces a holomorphic section in $E_{1}$. Let $\widehat{b}(t)$ be defined as above for the holomorphic sections $\widehat{\omega}_{1}, \widetilde{\omega}_{2}, \ldots, \widetilde{\omega}_{N}$. Then $b(t)=\left(t-t_{1}\right)^{2} \widehat{b}(t)$ and $\widehat{b}(t)$ is a rational function which is holomorphic in $B$. Continuing in this manner we obtain $N$ holomorphic sections $\varrho_{1}, \ldots, \varrho_{N} \in \mathcal{O}_{\mathrm{r}}\left(E_{1}\right)$ such that the corresponding rational function $\bar{b}(t)$ is a holomorphic nonvanishing function on $B$. Hence $\varrho_{1}, \ldots, \varrho_{N}$ form a basis in $\mathcal{O}_{\mathrm{r}}\left(E_{1}\right)$. The specific form of $\varrho_{1}, \ldots, \varrho_{N}$ follows from Theorem (3.4).

Let $\widetilde{\varrho}_{1}, \ldots, \widetilde{\varrho}_{N}$ be another basis in $\mathcal{O}_{\mathrm{r}}\left(E_{1}\right)$. Then (3.7) holds, with each $a_{i j}(t)$ a rational function which is holomorphic on $B$. Since $A(t)^{-1}$ has similar properties, $\operatorname{det} A(t)$ is a rational function which is holomorphic on $B$ and does not vanish there.

Assume that $\widetilde{\varrho}_{1}, \ldots, \widetilde{\varrho}_{N}$ are given by (3.7), where $A(t)$ has rational entries which are holomorphic in $B$. Then $\widetilde{\varrho}_{i} \in \mathcal{O}_{\mathrm{r}}\left(E_{1}\right)$. The assumption that $\operatorname{det} A(t)$ does not vanish on $B$ yields that $\widetilde{\varrho}_{1}, \ldots, \widetilde{\varrho}_{N}$ is a holomorphic basis in $\mathcal{O}_{\mathrm{r}}\left(E_{1}\right)$.

(3.8) Problem. Does there exist a basis $\varrho_{1}, \ldots, \varrho_{N}$ of $\mathcal{O}_{\mathrm{r}}\left(E_{1}\right)$ so that

$$
\varrho_{j}=p_{j} \frac{d g}{\operatorname{det} J(F)}, \quad p_{j} \in \mathbb{C}\left[\mathbb{C}^{2}\right], j=1, \ldots, N .
$$

Let $\varrho \in \mathcal{O}_{\mathrm{r}}\left(E_{1}\right)$. As we chose the Gauss-Manin connection, we deduce that $d \varrho_{t} / d t$ is a holomorphic section on $E_{1}$. The growth estimates (see e.g. [Mal]) yield that $d \varrho_{t} / d t \in \mathcal{O}_{\mathrm{r}}$. Let $\varrho_{1}, \ldots, \varrho_{N}$ be a holomorphic basis in $\mathcal{O}_{\mathrm{r}}\left(E_{1}\right)$. Then we obtain a Picard-Fuchs system:

$$
\frac{d x}{d t}=B(t) x, \quad x(t)=\left(\varrho_{1, t}, \ldots, \varrho_{N, t}\right)^{T}, B(t)=\left(b_{i j}(t)\right)_{i, j=1}^{N} .
$$

The entries of $B(t)$ are rational functions which are holomorphic on $B$. 
(3.10) Proposition. Let $f \in \mathbb{C}\left[\mathbb{C}^{2}\right]$ be a nonconstant primitive polynomial. Let $N=\operatorname{dim} H_{1}\left(V_{t}, \mathbb{Z}\right), t \in B$. Let $t_{0} \in B$ and choose a basis $\left[\gamma_{1}\right], \ldots,\left[\gamma_{N}\right]$ in $H_{1}\left(V_{t_{0}}, \mathbb{Z}\right)$ as in $\S 1$. Assume that $\varrho_{1}, \ldots, \varrho_{N}$ is a holomorphic basis in $\mathcal{O}_{\mathrm{r}}\left(E_{1}\right)$ such that

$$
\int_{\gamma_{i}\left(t_{0}\right)} \varrho_{j}=\delta_{i j}, \quad i, j=1, \ldots, N
$$

Consider the system

$$
\frac{d Y}{d t}=-B(t)^{T} Y, \quad Y\left(t_{0}\right)=I, \quad t \in B
$$

where $B(t)$ is the matrix induced by the Gauss-Manin connection given by (3.9). Then the analytic continuation of $Y\left(t_{0}\right)$ along $\tau \in \pi_{1}\left(B, t_{0}\right)$ gives the monodromy element $\phi_{1}^{*}(\tau)$.

Proof. Let $Y(t)=\left(y_{i j}(t)\right)_{i, j=1}^{N}$ and set

$$
\omega_{i, t}:=\sum_{j=1}^{N} y_{j i}(t) \varrho_{j, t}, \quad i=1, \ldots, N .
$$

Then $\omega_{i}$ describes a multivalued locally holomorphic section of $E_{1}$. Use (3.9) and the definition of $Y$ to deduce that $d \omega_{i, t} / d t=0, i=1, \ldots, N$. Thus $\omega_{1}, \ldots, \omega_{N}$ are locally constant holomorphic sections with respect to the Gauss-Manin connection. Note that the initial value $\omega_{1, t_{0}}, \ldots, \omega_{N, t_{0}}$ is the dual basis to the basis $\left[\gamma_{1}\right], \ldots,\left[\gamma_{N}\right]$. Hence the analytic continuation of $\omega_{1}, \ldots, \omega_{N}$ along $\tau$ will yield the dual basis to $\phi_{1}^{\prime}(\tau)\left(\left[\gamma_{1}\right]\right), \ldots, \phi_{1}^{\prime}(\tau)\left(\left[\gamma_{N}\right]\right)$.

Let $\omega$ be a rational 1-form on $\mathbb{C}^{2}$ which induces a rational section in $E_{1}$. Assume that $\omega_{t}$ is holomorphic in some open set $U \subset \mathbb{C}$. Fix $t \in U$ and a basis in $H_{1}\left(V_{t}, \mathbb{Z}\right)$ as in $\S 2$. Let

$$
\begin{aligned}
& c\left(\omega, \gamma_{j}(t)\right):=\int_{\gamma_{j}(t)} \omega_{t}, \quad j=1, \ldots, 2 \text { gen, } \\
& c^{1}(\omega, t):=\left(c\left(\omega, \gamma_{1}(t)\right), \ldots, c\left(\omega, \gamma_{2} \text { gen }(t)\right),\right. \\
& c\left(\omega, \gamma_{v, i}(t)\right)=\int_{\gamma_{v, i}(t)} \omega_{t}, \quad i=1, \ldots, k(v), v \in K, \\
& c(\omega, v, t):=\left(c\left(\omega, \gamma_{v, i}(t)\right)\right)_{1 \leq i \leq k(v)}, \\
& c^{2}(\omega, t):=(c(\omega, v, t))_{v \in V}, \quad c(\omega, t):=\left(c^{1}(\omega, t), c^{2}(\omega, t)\right) .
\end{aligned}
$$

Recall the equality (1.2) to deduce

$$
\sum_{v \in K} \sum_{i=1}^{k(v)} c\left(u, \gamma_{v, i}(t)\right)=0 .
$$


Observe

$$
\begin{aligned}
& c(p(f) \omega, t)=p(t) c(\omega, t), \quad p \in \mathbb{C}(\mathbb{C}), \\
& \omega=q \frac{d g}{\operatorname{det} J(F)}, \quad q \in \mathcal{F}\left[\mathbb{C}^{2}\right] .
\end{aligned}
$$

The following proposition is straightforward.

(3.13) Proposition. Let $f \in \mathbb{C}\left[\mathbb{C}^{2}\right]$ be a nonconstant primitive polynomial. Assume that $\omega$ is a rational 1 -form on $\mathbb{C}^{2}$ which induces a holomorphic section on $E_{1}$. Then $\omega_{t}, t \in B$ is a nontrivial fixed element under the action of the monodromy iff

$$
\frac{d}{d t} c(\omega, t)=0, \quad t \in B, \quad c(\omega, t) \not \equiv 0 .
$$

Problem (3.8) is closely related to the following problem. Let $\omega \in \Omega_{\mathrm{r}}^{1}\left(\mathbb{C}^{2}\right)$. When is $c(\omega, t) \equiv 0$ ? Clearly, this holds if $\omega$ is of the form

$$
\omega=d h+r d f=\left(h_{x}+r f_{x}\right) d x+\left(h_{y}+r f_{y}\right) d y, \quad h, r \in \mathbb{C}\left[\mathbb{C}^{2}\right] .
$$

That is, if $\omega$ is given by (3.1), when we can solve the system

$$
h_{x}=r f_{x}-p, \quad h_{y}=r f_{y}-q .
$$

The above system has a solution iff the following PDE is solvable:

$$
-f_{y} r_{x}+f_{x} r_{y}=-q_{x}+p_{y}
$$

for some $r \in \mathbb{C}\left[\mathbb{C}^{2}\right]$. This equation will be studied in the next section.

4. A differential operator. Let $F=\left(F_{1}, \ldots, F_{n}\right): \mathbb{C}^{n} \rightarrow \mathbb{C}^{n}$ be a polynomial map. Let $J(F)=\left(\partial F_{i} / \partial z_{j}\right)_{i, j=1}^{n}$ be the Jacobian matrix of $F$. Assume that $F$ is dominating, i.e. $\operatorname{det} J(F) \neq 0$. Let

$$
\operatorname{SPV}(F):=\left\{z \in \mathbb{C}^{n}: \operatorname{det} J(F)(z)=0\right\} .
$$

Let $\partial_{i}=\partial / \partial z_{i}, i=1, \ldots, n$, be the $n$ standard commuting vector fields on $\mathbb{C}^{n}$. We can pull back these abelian vector fields at all points $F\left(\mathbb{C}^{n}\right) \backslash$ $F(\operatorname{SPV}(F))$ to a set of $n$ rational commuting vector fields $D_{i}=F^{-1}\left(\partial_{i}\right)$, $i=1, \ldots, n$. More specifically, the chain rule yields the following formula for $D_{i}$ :

$$
D^{T}=\left(J(F)^{-1}\right)^{T} \partial^{T}, \quad D=\left(D_{1}, \ldots, D_{n}\right), \partial=\left(\partial_{1}, \ldots, \partial_{n}\right) .
$$

For $n=2$ we have

$$
\begin{aligned}
& D_{1}=\frac{1}{\operatorname{det} J(F)}\left(\frac{\partial F_{2}}{\partial z_{2}} \frac{\partial}{\partial z_{1}}-\frac{\partial F_{2}}{\partial z_{1}} \frac{\partial}{\partial z_{2}}\right), \\
& D_{2}=\frac{1}{\operatorname{det} J(F)}\left(-\frac{\partial F_{1}}{\partial z_{2}} \frac{\partial}{\partial z_{1}}+\frac{\partial F_{1}}{\partial z_{1}} \frac{\partial}{\partial z_{2}}\right) .
\end{aligned}
$$


Let

$$
L=\operatorname{det} J(F) D_{n} .
$$

Note that $L$ is a linear differential operator of first order with polynomial coefficients which depend only on $F_{1}, \ldots, F_{n-1}$. For $n=2$ the operator $-L$ is the standard Hamiltonian vector field associated with $F_{1}$ :

$$
L=-\frac{\partial F_{1}}{\partial z_{2}} \frac{\partial}{\partial z_{1}}+\frac{\partial F_{1}}{\partial z_{1}} \frac{\partial}{\partial z_{2}} .
$$

Note that the left-hand side of $(3.15)$ is $L(r)$ where $F_{1}=f$. $L$ is a nontrivial operator iff $F_{1}, \ldots, F_{n-1}$ are algebraically independent. In what follows we shall assume that $F_{1}, \ldots, F_{n-1}$ are algebraically independent. By picking a polynomial $F_{n}$ such that $F$ is a dominating map and using the above interpretation of $L$ we obtain

$$
L(v u)=v L u, \quad u \in \mathbb{C}\left(z_{1}, \ldots, z_{n}\right), v \in \mathbb{C}\left(F_{1}, \ldots, F_{n-1}\right) .
$$

Let $\mathcal{F}=\mathbb{C}\left(F_{1}, \ldots, F_{n-1}\right)$ be the field of rational functions in the $n-1$ variables $F_{1}(z), \ldots, F_{n-1}(z)$. Denote by $\mathcal{F}\left[\mathbb{C}^{n}\right] \subset \mathbb{C}\left(\mathbb{C}^{n}\right)$ the ring of polynomials in $z_{1}, \ldots, z_{n}$ with coefficients in $\mathcal{F}$. Then $u \in \mathcal{F}\left[\mathbb{C}^{n}\right]$ iff $u=p v$ where $p=p\left(z_{1}, \ldots, z_{n}\right)$ is a polynomial and $v \in \mathcal{F}$. Note that $\mathcal{F}\left[\mathbb{C}^{n}\right]$ is an infinite-dimensional vector space over the field $\mathcal{F}$ and the operator $L: \mathcal{F}\left[\mathbb{C}^{n}\right] \rightarrow \mathcal{F}\left[\mathbb{C}^{n}\right]$ is a linear operator over $\mathcal{F}$. Let $L\left(\mathcal{F}\left[\mathbb{C}^{n}\right]\right)$ be the range of $L$. In this section we show that $L$ is Fredholm.

Consider the map $\Phi=\left(F_{1}, \ldots, F_{n-1}\right): \mathbb{C}^{n} \rightarrow \mathbb{C}^{n-1}$. Recall that $z$ is called a singular point for $\Phi$ if $\operatorname{rank} J(\Phi)<n-1 . \xi \in \mathbb{C}^{n-1}$ is called a singular value if $\xi=\Phi(z)$ for some singular point $z$. Clearly, the set of all singular points is a closed variety of codimension at least one. Sard's theorem (see e.g. [Mil]) yields that the set of all singular values is a closed subvariety of codimension at least one. Moreover, as in the case $n=2$, there exists a closed proper subvariety $\operatorname{CVV}(\Phi) \subset \mathbb{C}^{n-1}$ with the following property: For any $\xi \in \mathbb{C}^{n-1} \backslash \operatorname{CVV}(\Phi)$, the fiber $\Phi^{-1}(\xi)$ is a union of $d$ smooth irreducible curves, each homeomorphic to a fixed compact Riemann surface $\Sigma$ punctured at $k$ points. We call these fibers regular. If $d=1, \Phi$ is called primitive. If $d>1$, one can show that there exists a dominating polynomial map $H: \mathbb{C}^{n-1} \rightarrow \mathbb{C}^{n-1}$, with $\operatorname{deg}(H)=d$ and a primitive polynomial map $\Psi: \mathbb{C}^{n} \rightarrow \mathbb{C}^{n-1}$ so that $\Phi=H \circ \Psi$. (We are not going to use this fact.) In what follows we assume that $\Phi$ is primitive. Let $a \in \mathbb{C}^{n}$ be a regular point of $\Phi$. Set $\xi=\Phi(a)$. Then there exists a unique fiber $V_{\xi}=\Phi^{-1}(\xi)$ which passes through $a$. Furthermore, $a$ is a smooth point of $V_{\xi}$. Define a local 1 -form on $V_{\xi}$ in the neighborhood of $a$ as follows. Consider the $n-1$ 1-forms $d F_{1}, \ldots, d F_{n-1}$. They are linearly independent in the neighborhood of $a$. Let $\theta=\sum_{i=1}^{n} \theta_{i} d z_{i}$ be an analytic 1-form in the neighborhood of $a$ so that

$$
d F_{1} \wedge \ldots \wedge d F_{n-1} \wedge \theta=d z_{1} \wedge \ldots \wedge d z_{n} .
$$


Define $\alpha$ to be the restriction of $\theta$ to $V_{\xi}$ in the neighborhood of $a$. It is straightforward to show that $\alpha$ is defined uniquely, independently of the choice of $\theta$. In particular, if $F_{n}$ is algebraically independent of $F_{1}, \ldots, F_{n-1}$ and $a \notin \operatorname{SPV}(F)$, then

$$
\alpha=\operatorname{det} J(F)^{-1} d F_{n} \mid V_{\xi}
$$

in the neighborhood of $a$.

(4.2) Theorem. Let $\Phi=\left(F_{1}, \ldots, F_{n-1}\right): \mathbb{C}^{n} \rightarrow \mathbb{C}^{n-1}$ be a primitive dominating polynomial map. For a Zariski open set $T \subset \mathbb{C}^{n-1}$ of regular fibers $V_{\xi}=\Phi^{-1}(\xi), \xi \in \mathbb{C}^{n-1}$, let $\alpha$ be a 1-form defined by (4.1) for some $F_{n} \in \mathbb{C}\left[\mathbb{C}^{n}\right]$ such that $F=\left(F_{1}, \ldots, F_{n}\right)$ is dominating. Assume that $f \in \mathcal{F}\left[\mathbb{C}^{n}\right]$. Then $f \in L\left(\mathcal{F}\left[\mathbb{C}^{n}\right]\right)$ iff

$$
\int_{\gamma} f \alpha=0
$$

for any closed path $\gamma \subset V_{\xi}, \xi \in T$.

Pr o of. Assume first that $L u=f$ for some $u \in \mathcal{F}\left[\mathbb{C}^{n}\right]$. By multiplying $u$ and $f$ by an appropriate polynomial $p\left(F_{1}, \ldots, F_{n-1}\right)$ it is enough to assume that $u, f$ are polynomials. We claim that the restriction of $d u$ to any regular fiber $V_{\xi}$ is $f \alpha$. Indeed, let $a \in V_{\xi}$ with $\operatorname{det} J(F)(a) \neq 0$. Let $w=F(z)$. Then

$$
d u=\sum_{i=1}^{n} \frac{\partial u}{\partial w_{i}} d w_{i}
$$

The restriction of $d u$ to $w_{i}=\xi_{i}, i=1, \ldots, n-1$, yields

$$
d u=\frac{\partial u}{\partial w_{n}} d w_{n}=L u \frac{d F_{n}}{\operatorname{det} J(F)}=f \alpha .
$$

Hence the condition of the theorem is necessary.

We now prove the sufficiency. Pick a linear function $\lambda$ on $\mathbb{C}^{n}$ such that the hyperplane $\lambda=t_{0}$ intersects a Zariski open set of regular fibers $V_{\xi}$ at $d$ distinct points $\zeta_{1}(\xi), \ldots, \zeta_{d}(\xi)$. Assume that $V_{\xi}$ is a regular fiber such that $f \mid V_{\xi}$ is holomorphic, the condition (4.3) holds and $\lambda=t_{0}$ intersects $V_{\xi}$ at $d$ distinct points. Hence there exists $u \in \mathcal{O}_{\mathrm{r}}\left(V_{\xi}\right)$ so that $d u=f \alpha$. Fix $u$ uniquely by the condition

$$
\sum_{i=1}^{d} u\left(\zeta_{i}(\xi)\right)=0 .
$$

Then $u$ is analytic on $\mathbb{C}^{n} \backslash S$, where $S$ is the zero set of some polynomial $p\left(F_{1}, \ldots, F_{n-1}\right)$. Clearly, $L u=f$ on $\mathbb{C}^{n} \backslash S$. By construction, the singularities of $u$ are rational, i.e. $u \in \mathcal{F}\left[\mathbb{C}^{n}\right]$. 
(4.4) Theorem. Let $\Phi=\left(F_{1}, \ldots, F_{n-1}\right): \mathbb{C}^{n} \rightarrow \mathbb{C}^{n-1}$ be a primitive dominating polynomial map. Then $L: \mathcal{F}\left[\mathbb{C}^{n}\right] \rightarrow \mathcal{F}\left[\mathbb{C}^{n}\right]$ is Fredholm. More precisely,

$$
\operatorname{dim} \operatorname{ker}(L)=1, \quad \operatorname{dim} \mathcal{F}\left[\mathbb{C}^{n}\right] / L\left(\mathcal{F}\left[\mathbb{C}^{n}\right]\right)=\operatorname{dim} \mathcal{H}^{1}(V),
$$

where $V$ is any regular fiber $\Phi^{-1}(\xi)$.

Proof. Assume that $L(u)=0$ and $u \in \mathcal{F}\left[\mathbb{C}^{n}\right]$. Multiply $u$ by an appropriate polynomial in $F_{1}, \ldots, F_{n-1}$ to obtain a polynomial $v$ so that $L(v)=0$. Choose $F_{n}$ so that $\left(F_{1}, \ldots, F_{n}\right)$ is dominating. Then $v$ is algebraic in $F_{1}, \ldots, F_{n-1}$. As $v$ is a polynomial, it follows that $v=q\left(F_{1}, \ldots, F_{n-1}\right)$. Thus $u \in \mathcal{F}$ and $\operatorname{ker}(L)$ is the one-dimensional space over $\mathcal{F}$ spanned by the function 1 .

We now prove the second part of the theorem. Pick a regular fiber $V$. Let $N=\operatorname{dim} \mathcal{H}^{1}(V)$. Choose a basis $\omega_{1}, \ldots, \omega_{N} \in \mathcal{H}^{1}(V)$. Then $\omega_{i} / \alpha=$ $p_{i} \in \mathbb{C}\left[\mathbb{C}^{n}\right], i=1, \ldots, N$. Hence the forms $p_{i} \alpha, i=1, \ldots, N$, span $\mathcal{H}^{1}\left(V_{\xi}\right)$, for a Zariski open set of regular fibers $V_{\xi}$. Theorem (4.2) yields that

$$
L(u)=\sum_{i=1}^{N} a_{i} p_{i}, \quad a_{i} \in \mathcal{F}, i=1, \ldots, N,
$$

is not solvable in $\mathcal{F}\left[\mathbb{C}^{n}\right]$ unless $a_{1}=\ldots=a_{N}=0$. Hence $\operatorname{dim} \operatorname{coker}(L) \geq N$. We now show that $\operatorname{dim} \operatorname{coker}(L) \leq N$. Let $f \in \mathcal{F}\left[\mathbb{C}^{n}\right]$. Assume that $V_{\xi}$ is a regular fiber so that $f$ is analytic on $V_{\xi}$ and $p_{1} \alpha, \ldots, p_{N} \alpha$ span $\mathcal{H}^{1}\left(V_{\xi}\right)$. Then there exist unique $a_{1}(\xi), \ldots, a_{m}(\xi) \in \mathbb{C}$ so that

$$
\left(f+\sum_{i=1}^{N} a_{i}(\xi) p_{i}\right) \alpha \in d \mathcal{O}_{\mathrm{r}}\left(V_{\xi}\right) .
$$

Since $f \in \mathcal{F}\left[\mathbb{C}^{n}\right]$ and $p_{1}, \ldots, p_{N}$ are polynomials, we deduce that $a_{i}(\xi)$, $i=1, \ldots, N$, are rational functions in $n-1$ variables. Then

$$
g=f+\sum_{i=1}^{N} a_{i}\left(F_{1}, \ldots, F_{n-1}\right) p_{i}
$$

satisfies the condition of Theorem (4.2). Thus there exists $u \in \mathcal{F}\left[\mathbb{C}^{n}\right]$ so that $L u=g$. Hence dim $\operatorname{coker}(L)=N$.

Consider the example $f=x^{2} y$. Then for $t \neq 0$ the fiber $f=t$ is a complex plane punctured at the origin with coordinate $x$. Hence $H_{1}\left(V_{t}, \mathbb{Z}\right)=\mathbb{Z}$. As $f=0$ is reducible it follows that $\mathrm{CVV}(f)=\{0\}$. Clearly

$$
L=-x^{2} \frac{\partial}{\partial x}+2 x y \frac{\partial}{\partial y} .
$$

Let $g=x$. Then $\alpha=-d x / x^{2}$. As $x \alpha$ has a nontrivial residue on $\mathbb{C} \backslash\{0\}$, Theorem (4.2) yields that $x \notin L\left(\mathcal{F}\left[\mathbb{C}^{2}\right]\right)$. Theorem (4.4) implies that $\mathcal{U}:=$ 
$\mathcal{F}\left[\mathbb{C}^{2}\right] / L\left(\mathcal{F}\left[\mathbb{C}^{2}\right]\right)$ is one-dimensional. We now show explicitly that $x$ is a basis in $\mathcal{U}$. Let $p(x, y) \in \mathcal{F}\left[\mathbb{C}^{2}\right]$. Set

$$
\begin{aligned}
q(x, t) & :=p\left(x, t x^{-2}\right)=\sum_{i=-m}^{n} \alpha_{i}(t) x^{i}, \quad \alpha_{i} \in \mathbb{C}(\mathbb{C}), i=-m, \ldots, n, \\
q_{1}(x, t) & :=\sum_{-m \leq i \leq n, i \neq 1}-\alpha_{i}(t) \frac{x^{i-1}}{i-1}, \quad q_{2}(x, y):=q_{1}\left(x, x^{2} y\right) .
\end{aligned}
$$

As $x^{-k}=(x y / f)^{k}, k \geq 1$, it follows that $q_{2}(x, y) \in \mathcal{F}\left[\mathbb{C}^{2}\right]$. The equalities $L(f)=0$ and $L(x)=-x^{2}$ yield $p(x, y)-\alpha_{1}(f) x=L\left(q_{2}(x, y)\right)$. Hence $x$ spans $\mathcal{U}$.

Compare Theorems (3.4) and (4.4) to deduce:

(4.5) Corollary. Let $F_{1}=f \in \mathbb{C}\left[\mathbb{C}^{2}\right]$ be a nonconstant primitive polynomial. Then $\mathcal{R}\left(E_{1}\right)$ is isomorphic to

$$
\mathcal{U}:=\mathcal{F}\left[\mathbb{C}^{2}\right] / L\left(\mathcal{F}\left[\mathbb{C}^{2}\right]\right) .
$$

More precisely, two rational forms $p d g / \operatorname{det} J(F), q d g / \operatorname{det} J(F), p, q \in$ $\mathcal{F}\left[\mathbb{C}^{2}\right]$, induce the same section in $\mathcal{R}\left(E_{1}\right)$ iff $p-q \in L\left(\mathcal{F}\left[\mathbb{C}^{2}\right]\right)$.

Let $f \in \mathbb{C}\left[\mathbb{C}^{2}\right]$ be a nonconstant polynomial with no critical points, i.e. every affine fiber $V_{t}$ is smooth. For example,

$$
f=x+x^{m} y^{n}, \quad m, n \geq 1 .
$$

Let $L$ be the operator associated with $f$ and consider the equation

$$
L(u)=h, \quad h \in \mathbb{C}\left[\mathbb{C}^{2}\right] .
$$

Note that (3.15) is of the above form. For a given point $\zeta \in \mathbb{C}^{2}$ it is possible to find a linear function $g=a x+b y$ so that $\operatorname{det} J(F)(\zeta) \neq 0$ and $F=(f, g)$. Pushing (4.7) to the image plane $(u, v)=(f, g)$ we deduce that (4.7) is locally solvable at any point $\zeta$. Assume that $u_{i} \in \mathcal{O}_{i}, i=1,2$, are two local solutions in two open sets $O_{1}, O_{2}$ with nontrivial intersection. (Here $\mathcal{O}_{i}$ is the ring of holomorphic functions in $O_{i}$.) Then $L\left(u_{1}-u_{2}\right)=0$ in $O_{1} \cap O_{2}$. It is straightforward to deduce that $u_{1}-u_{2}=\phi(f)$ in $O_{1} \cap O_{2}$ for some holomorphic function $\phi$ on $f\left(O_{1} \cap O_{2}\right) \subset \mathbb{C}$. Let $\mathcal{O}_{f}$ be the pull back of the sheaf of holomorphic functions on $\mathbb{C}$ by the map $f: \mathbb{C}^{2} \rightarrow \mathbb{C}$. Then the obstruction to patching together the local solutions of $(4.7)$ is $H^{1}\left(\mathbb{C}^{2}, \mathcal{O}_{f}\right)$ (see $[\mathrm{G}-\mathrm{H}]$ ). Our results show that

$$
\operatorname{rank} H^{1}\left(\mathbb{C}^{2}, \mathcal{O}_{f}\right) \geq \operatorname{dim} \mathcal{H}^{1}\left(V_{t}\right),
$$

for a regular fiber $V_{t}$. (We do not know if the above inequality is sharp.) For the example (4.6) it follows that $\operatorname{dim} \mathcal{H}^{1}\left(V_{t}\right) \geq 1$, as $V_{t}, t \neq 0$, is a compact Riemann surface punctured at two points at least. Hence for some $h \in \mathbb{C}\left[\mathbb{C}^{2}\right],(4.7)$ does not have a global holomorphic solution. 
5. The plane Jacobian conjecture. Let $F=\left(F_{1}, \ldots, F_{n}\right): \mathbb{C}^{n} \rightarrow \mathbb{C}^{n}$ be a polynomial map. Assume that $F$ is a local diffeomorphism. Then $\operatorname{det} J(F)=$ const $\neq 0$. The Jacobian conjecture claims that $F$ is a diffeomorphism. Then the form $\alpha$ defined in (4.1) is given globally by $d F_{n} / \operatorname{det} J(F)$. In this case the operators $D_{1}, \ldots, D_{n}$ were studied in [N-S]. In what follows we study the plane Jacobian conjecture $(n=2)$. We assume that $F=(f, g): \mathbb{C}^{2} \rightarrow \mathbb{C}^{2}$ is a local polynomial diffeomorphism. Furthermore, we assume the normalization $J(F)(0)=I$. In particular, det $J(F)=1$. Let $L=D_{2}, M=D_{1}$ be the first order differential operators defined in $\S 4$. Note

$$
L(g)=M(f)=1, \quad L(f)=M(g)=0 .
$$

The following theorem is due to T. Krasiński and S. Spodzieja [K-S, Thm. 4.1]. (Theorem 4.1 of $[\mathrm{K}-\mathrm{S}]$ holds for any $n \geq 2$.) An earlier analytic version of this theorem for $n=2$ is proved in [Ste2]. We recall its proof for $n=2$ for convenience:

(5.1) Theorem. Let $F: \mathbb{C}^{2} \rightarrow \mathbb{C}^{2}$ be a local polynomial diffeomorphism. Then $F$ is a diffeomorphism iff coker $L$ is trivial.

Pr o of. Clearly, if $F$ is a diffeomorphism then coker $L$ is trivial. Assume that $F$ is a local diffeomorphism and coker $L=0$. As $f$ does not have critical points, $f$ is primitive. According to Theorem (4.4), $\mathcal{H}^{1}\left(V_{t}\right)=0$ for a regular fiber $V_{t}=f^{-1}(t)$. Hence $V \sim \mathbb{C}$. As the map $g: V \rightarrow \mathbb{C}$ is a local diffeomorphism it follows that it is a diffeomorphism. Hence $\operatorname{deg}(F)=1$ and $F$ is a diffeomorphism.

We call $F$ a Jacobian pair if $F$ is a local diffeomorphism which is not a diffeomorphism. Theorems (2.5) and (2.11) yield:

(5.2) Corollary. Let $F=(f, g)$ be a Jacobian pair. Assume that each fiber $V_{t}$ is irreducible. Then the monodromy action on the cohomology $\mathcal{H}^{1}\left(V_{t}\right)$ does not have a nontrivial fixed element. In particular, the genus of the closure $\Sigma_{t}$ of the regular fiber $V_{t}$ is at least two.

The inequality part of Corollary (5.2) was proved by Razar [Raz] (gen $>1)$, Heitmann [Hei] and Lê and Weber $[\mathrm{L}-\mathrm{W}]($ gen $>0)$. Assume that $F$ is a Jacobian pair. Then Kaliman [Kal] showed that there exists a plane polynomial diffeomorphism $G$ so that for $G \circ F:=\left(f_{1}, g_{1}\right)$ all the fibers $f_{1}^{-1}(t)$ are irreducible.

In what follows we assume that $F$ is a Jacobian pair unless otherwise stated. Let $u \in \mathbb{C}\left[\mathbb{C}^{2}\right]$. Then a necessary condition for the existence of a solution $L(v)=u, v \in \mathbb{C}\left[\mathbb{C}^{2}\right]$, is given by Theorem (4.2). In view of Theorem (5.1) there exist $u$ for which $L(v)=u$ is not solvable. However, the following result holds. 
(5.3) Proposition. Let $F=(f, g)$ be a Jacobian pair. Then for any $u \in$ $\mathbb{C}\left[\mathbb{C}^{2}\right]$ there exist $v, w \in \mathbb{C}\left[\mathbb{C}^{2}\right]$ so that $L(v)+M(w)=u$.

Proof. Let $s(x, y)=\int_{0}^{x} u(t, y) d t$. Then $u d x \wedge d y=d(s d y)$. Observe that for any $h \in \mathbb{C}\left(\mathbb{C}^{2}\right)$ we have $d h=L(h) d g+M(h) d f$. Hence

$$
\begin{aligned}
d y & =L(y) d g+M(y) d f=f_{x} d g-g_{x} d f, \\
d(s d y) & =d\left(s f_{x} d g-s g_{x} d f\right)=\left(M\left(s f_{x}\right)+L\left(s g_{x}\right)\right) d f \wedge d g \\
& =\left(M\left(s f_{x}\right)+L\left(s g_{x}\right)\right) d x \wedge d y, \\
u & =M\left(s f_{x}\right)+L\left(s g_{x}\right) .
\end{aligned}
$$

(5.4) Lemma. Let $F=(f, g)$ be a Jacobian pair. Let $u \in \mathcal{F}\left[\mathbb{C}^{2}\right]$ and assume that $u$ is analytic on each $V_{t}$, where $t$ varies in $D\left(t_{0}, \varepsilon\right)$ (disk of radius $\varepsilon$ centered at $\left.t_{0}\right)$. Let $\gamma\left(t_{0}\right) \subset V_{t_{0}}$ be a closed smooth curve which extends continuously to a family of smooth closed curves in $V_{t}, t \in D\left(t_{0}, \varepsilon\right)$. Then

$$
\frac{d}{d t} \int_{\gamma(t)} u d g=\int_{\gamma(t)} M(u) d g .
$$

In particular

$$
\frac{d}{d t} c(u d g, t)=c(M(u) d g, t), \quad u \in \mathcal{F}\left[\mathbb{C}^{2}\right]
$$

$(\operatorname{see}(3.11))$.

Proof. The closed smooth curve $F\left(\gamma\left(t_{0}\right)\right) \subset \mathbb{C}^{2}$ is of the form $\left(t_{0}, \delta\left(t_{0}\right)\right)$, where $\delta\left(t_{0}\right)$ is the projection of $F\left(\gamma\left(t_{0}\right)\right)$ on the second coordinate. Let $\mu(t):=\left(t, \delta\left(t_{0}\right)\right) \subset \mathbb{C}^{2}$ be the closed curve whose projection on the first coordinate is $t$ and on the second coordinate is the closed curve $\delta\left(t_{0}\right)$. Since $F$ is a local diffeomorphism it follows that there exists $\varepsilon^{\prime}, 0<\varepsilon^{\prime}<\varepsilon$, such that for each $t \in D\left(t_{0}, \varepsilon^{\prime}\right)$ there exists a smooth closed path $\delta^{\prime}(t)$, depending continuously on $t$, which satisfies

$$
\delta^{\prime}(t) \subset V_{t}, \quad F\left(\delta^{\prime}(t)\right)=\mu(t), \quad \delta^{\prime}\left(t_{0}\right)=\gamma\left(t_{0}\right) .
$$

Clearly, $\left[\delta^{\prime}(t)\right]=[\gamma(t)] \in H_{1}\left(V_{t}, \mathbb{Z}\right), t \in D\left(t_{0}, \varepsilon^{\prime}\right)$. Hence

$$
\int_{\gamma(t)} u d g=\int_{\delta^{\prime}(t)} u d g=\int_{s \in \delta\left(t_{0}\right)} \widetilde{u}(t, s) d s .
$$

Here $\widetilde{u}$ is a multivalued algebraic function on $\mathbb{C}^{2}$ obtained by pushing forward $u$ using $F$. Since the branches of $\widetilde{u}$ appearing in the above integral do not have singular points in the neighborhood of $\mu\left(t_{0}\right)$, we deduce 


$$
\begin{aligned}
\frac{\partial}{\partial t} \int_{s \in \delta\left(t_{0}\right)} \widetilde{u}(t, s) d s & =\int_{s \in \delta\left(t_{0}\right)} \frac{\partial}{\partial t} \widetilde{u}(t, s) d s=\int_{\delta^{\prime}(t)} M(u) d g \\
& =\int_{\gamma(t)} M(u) d g .
\end{aligned}
$$

In particular, (5.5) holds.

(5.6) Corollary. Let $F=(f, g)$ be a Jacobian pair. Let

$$
\mathcal{N}:=\left\{c(\omega, t): \omega \in \Omega_{\mathrm{r}}^{1}\left(\mathbb{C}^{2}\right), t \in B\right\} .
$$

Then $\mathcal{N}$ is a $\mathbb{C}[t]$-module which satisfies the equality $\frac{d}{d t} \mathcal{N}=\mathcal{N}$.

Proof. (3.11)-(3.12) show that $\mathcal{N}$ is a $\mathbb{C}[t]$-module. Let $\omega=p d x+$ $q d y, p, q \in \mathbb{C}\left[\mathbb{C}^{2}\right]$. As $\operatorname{det} J(F)=1$ we deduce $\omega=u d g+u_{1} d f, u, u_{1} \in$ $\mathbb{C}\left[\mathbb{C}^{2}\right]$. Clearly, $c(\omega, t)=c(u d g, t)$. Use Proposition (5.3), Theorem (4.2) and Lemma (5.4) to deduce

$$
\begin{aligned}
c(\omega, t) & =c(u d g, t)=c(L(v) d g+M(w) d g, t) \\
& =c(M(w) d g, t)=\frac{d}{d t} c(w d g, t) .
\end{aligned}
$$

(5.7) Proposition. Let $F=(f, g)$ be a Jacobian pair. Consider the isomorphism $\iota: \mathcal{R}\left(E_{1}\right) \rightarrow \mathcal{U}$ given in Corollary (4.5). Then the following diagram commutes:

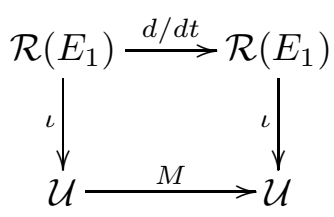

That is,

$$
\frac{d}{d t}(u d g)_{t}=(M(u) d g)_{t}, \quad u \in \mathcal{F}\left[\mathbb{C}^{2}\right], t \in B .
$$

Proof. As $M L=L M$ it follows that $M: \mathcal{U} \rightarrow \mathcal{U}$ is a linear differential operator on $\mathcal{U}$ :

$$
\begin{array}{r}
M(a(f) u+b(f) v)=a(f) M(u)+b(f) M(v)+a^{\prime}(f) u+b^{\prime}(f) v \\
a(t), b(t) \in \mathbb{C}(t), u, v \in \mathcal{U} .
\end{array}
$$

Let $p_{1}, \ldots, p_{N} \in \mathbb{C}\left[\mathbb{C}^{2}\right]$ be a basis in $\mathcal{U}$. Choose $t_{0} \in B$ and let $a_{1}, \ldots, a_{N}$ be holomorphic functions in an open set $U \subset B$ with $t_{0} \in U$. Let $u:=$ $\sum_{i=1}^{N} a_{i}(f) p_{i}$. Then $u$ is holomorphic on $U^{\prime}:=\bigcup_{t \in U} V_{t}$. Suppose that $(u d g)_{t}$, $t \in U$, gives a constant section in $\mathcal{O}\left(E_{1}\right)(U)$, i.e. $\frac{d}{d t}(u d g)_{t}=0$. From the definition of the Gauss-Manin connection it follows that $c(u d g, t)$ is a constant vector on $U$. Use the arguments of Lemma (5.4) to deduce the validity of (5.5) in this case, i.e. $c(M(u) d g, t)=0$. Vice versa, if $u \in \mathcal{O}\left(U^{\prime}\right)$ and 
$c(M(u) d g, t)=0$ then $(u d g)_{t}$ is a holomorphic constant section of $\mathcal{O}\left(E_{1}\right)(U)$. Combine (3.12) and (5.9) to deduce (5.8).

View $\mathcal{V}^{\prime}:=\mathbb{C}\left[\mathbb{C}^{2}\right] \cap L(\mathcal{F}[x, y])$ as a module over the ring $\mathcal{K}:=\mathbb{C}[f]$. Let $\mathcal{V}$ be the quotient module $\mathbb{C}[x, y] / \mathcal{V}^{\prime}$. Clearly, $\mathcal{F}$ is the field generated by $\mathcal{K}$ and $\mathcal{U}=\mathcal{V} \otimes \mathcal{F}$. Moreover, $M: \mathcal{V} \rightarrow \mathcal{V}$ is a differential operator over $\mathcal{K}$. Corollary (5.6) is equivalent to

$$
M(\mathcal{V})=\mathcal{V} .
$$

(5.11) TheOrem. Let $F=(f, g)$ be a Jacobian pair. Then the monodromy action on the cohomology $\mathcal{H}^{1}\left(V_{t}\right)$ of the regular fiber $V_{t}$ has a nontrivial fixed element iff the operator $M: \mathcal{U} \rightarrow \mathcal{U}$ is not injective.

Proof. Assume first that $M(u)=0$ for some $0 \neq u \in \mathcal{U}$. Suppose that $u$ is represented by $v \in \mathcal{F}\left[\mathbb{C}^{2}\right] \backslash L\left(\mathcal{F}\left[\mathbb{C}^{2}\right]\right)$. Proposition (5.7) shows that $v d g$ induces a nonzero constant section of $\mathcal{O}_{\mathrm{r}}\left(E_{1}\right)$. This section represents a nontrivial fixed element under the action of monodromy.

Assume now that $\phi \in \mathcal{H}^{1}\left(V_{t_{0}}\right), t_{0} \in B$, is a nontrivial invariant element under the monodromy action. Let $\phi_{t} \in \mathcal{H}^{1}\left(V_{t}\right), t \in B$, be the continuation of $\phi$ using the monodromy action. Pick a basis $\varrho_{1}, \ldots, \varrho_{N}$ in $\mathcal{O}_{\mathrm{r}}\left(E_{1}\right)$. Then

$$
\phi_{t}=\sum_{i=1}^{N} e_{i}(t) \varrho_{i, t}, \quad e_{1}, \ldots, e_{N} \in \mathcal{O}(B) .
$$

We claim that $e_{1}, \ldots, e_{N}$ are meromorphic on $\operatorname{CVV}(f) \cup\{\infty\}$. Use the arguments of the proof of Proposition (3.10) to deduce that $e:=\left(e_{1}, \ldots, e_{N}\right)^{T}$ satisfies the differential equation $d e / d t=-B(t)^{T} e$. Recall that a local change of basis around $t_{1} \in \mathrm{CVV}(f) \cup\{\infty\}$ will replace the system (3.9) by a matrix $\widetilde{B}(t)$ which has at most a pole at $t_{1}$ ([Del1]). In this new basis the equation $d \widetilde{e} / d t=-\widetilde{B}(t)^{T} \widetilde{e}$ has a regular singular point. Hence $\widetilde{e}$ and $e$ are meromorphic at $t_{1}$. Thus $e_{1}(t), \ldots, e_{N}(t)$ are rational functions. The 1-form $\psi:=\sum_{i=1}^{N} e_{i}(f) \varrho_{i}$ induces the constant section $\phi_{t}=\psi_{t}$. Use Proposition (5.7) to deduce the existence of $0 \neq u \in \mathcal{U}$ so that $M(u)=0$.

Let $p_{1}, \ldots, p_{N} \in \mathcal{F}\left[\mathbb{C}^{2}\right]$ be a basis in $\mathcal{U}$. Then

$$
\begin{gathered}
M\left(p_{i}\right) d g-\sum_{j=1}^{N} b_{i j}(f) p_{j} d g \in L\left(\mathcal{F}\left[\mathbb{C}^{2}\right]\right), \\
b_{i j}(t) \in \mathbb{C}(\mathbb{C}), i, j=1, \ldots, N .
\end{gathered}
$$

Suppose furthermore that $\varrho_{1}:=p_{1} d g, \ldots, \varrho_{N}:=p_{N} d g$ induces a basis in $\mathcal{O}_{\mathrm{r}}\left(E_{1}\right)$. Then the matrix $B(t)=\left(b_{i j}(t)\right)_{i, j=1}^{N}$ is given in (3.9). For a simply connected open set $U \subset B$ choose a basis in $H_{1}\left(V_{t}, \mathbb{Z}\right)$ which depends 
continuously on $t$ as in $\S 2$. Set

$$
x_{i j}(t)=\int_{\gamma_{j}(t)} p_{i} d g, \quad i, j=1, \ldots, N, t \in U, X=\left(x_{i j}(t)\right)_{i, j=1}^{N} .
$$

Lemma (5.4) yields that

$$
\frac{d}{d t} X=B(t) X(t)
$$

for $t \in U$. In fact, (5.14) is dual to the system given in Proposition (3.10). $\left(Y(t)^{T} X(t)\right.$ is a constant matrix.) Let $\tau \in \pi_{1}\left(B, t_{0}\right)$. Continue the solution of (5.14) using the path $\tau$ to obtain $X_{\tau}\left(t_{0}\right)$. Then

$$
\begin{gathered}
A(\tau)=\left(a_{i j}(\tau)\right)_{i, j=1}^{N}:=\phi_{1}^{\prime}(\tau), \\
\int_{\phi_{1}(\tau)\left(\gamma_{j}\left(t_{0}\right)\right)} p_{i} d g=\sum_{n=1}^{N} a_{n j}(\tau) \int_{\gamma_{n}\left(t_{0}\right)} p_{i} d g, \quad i, j=1, \ldots, N .
\end{gathered}
$$

Hence

$$
X_{\tau}\left(t_{0}\right)=X\left(t_{0}\right) A(\tau), \quad \tau \in \pi_{1}\left(B, t_{0}\right) .
$$

Recall that the system (5.14) at any singular point $t_{1}$ can be reduced locally to a system with a regular singular point at $t_{1}$ ([Del1]).

Let $R$ (gen, 0 ) be the moduli space of all compact orientable closed Riemann surfaces of genus gen. Consider the holomorphic map $\theta: B \rightarrow R$ (gen, 0 ) given by $t \mapsto\left[R\left(\Sigma_{t}\right)\right]$ (see $\S 1$ ). According to [Gri, §13], $\theta$ has at most a logarithmic singularity at each singular point of (5.14).

Let $F=(f, g)$ be a Jacobian pair. Let $\breve{F}$ be the minimal resolution given in Theorem (1.6). According to Proposition (1.7), $X:=\check{F}^{-1}(\mathbb{C} \times \mathbb{C})$ is a Stein manifold. Hence $X$ can be (properly) embedded as an affine smooth algebraic variety $X_{1} \subset \mathbb{C}^{n}$ for some $n>2$. It is known that $X$ can be properly embedded as a Stein manifold $Y \subset \mathbb{C}^{4}$ (see [For2]). See also [B-N, $\S 3]$. Furthermore, $X$ embeds in $\mathbb{C}^{3}$ iff $X$ is parallelizable (as a complex manifold) [For1].

(5.16) Theorem. Let $F=(f, g)$ be a Jacobian pair. Assume that each affine fiber $f=t$ is irreducible. Let $\bar{F}$ be the minimal resolution of $F$ given by Theorem (1.6). Then the Stein manifold $X=\check{F}^{-1}(\mathbb{C} \times \mathbb{C})$ is not parallelizable.

Proof. Assume to the contrary that $X$ is parallelizable. Then there exists a proper embedding $\iota: X \rightarrow \mathbb{C}^{3} \cdot \iota(X)$ is a two-dimensional connected Stein manifold in $\mathbb{C}^{3}$. As $H^{q}\left(\mathbb{C}^{3}, \mathcal{O}^{*}\right)=0$ for $q>0$, there exists $h \in \mathcal{O}\left(\mathbb{C}^{3}\right)$ such that $\iota(X)=Y$ is the zero set of $h$. Since $\iota: X \rightarrow Y$ is a proper embedding, $h$ does not have critical points on $Y$. Let $\check{V}_{t}:=\check{f}^{-1}(t) \subset X$, $t \in B$. Let $B^{\prime} \subset B$ be all the regular values of $\check{f}: X \rightarrow \mathbb{C}$. Clearly, $B \backslash B^{\prime}$ is a finite set. Note that $V_{t} \subset \check{V}_{t}$ and $\check{g}_{t}: \check{V}_{t} \rightarrow \mathbb{C}$ is a proper map of a 
fixed degree $d>1$ for $t \in B^{\prime}$. In fact, $\check{V}_{t}$ can be viewed as follows. Let $\widetilde{f}: M \rightarrow \mathbb{C P}^{1}$ be the minimal resolution described in Corollary (1.5). Let $\widetilde{g}: M \rightarrow \mathbb{C P}^{1}$ be the lifting of $g: \mathbb{C}^{2} \rightarrow \mathbb{C}$. For each $\widetilde{l}_{v}, v \in K$, we have three possibilities. The first one is that $\widetilde{g}$ is holomorphic on $\widetilde{l}_{v}$ and $\widetilde{g}(z)=\infty$ for all $z \in \widetilde{l}_{v}$. Then for each $t \in \mathbb{C}$ the points $\zeta_{v, 1}(t), \ldots, \zeta_{v, k(v)}(t)$ are not in $X$. The second possibility is that $\widetilde{g}$ is holomorphic on $\widetilde{l}_{v} \backslash T_{v}$ and $\widetilde{g}(z)=\infty$ for all $z \in \widetilde{l}_{v} \backslash T_{v}$. Here $T_{v}$ is a finite set. Then $\zeta_{v, 1}(t), \ldots, \zeta_{v, k(v)}(t), t \in \mathbb{C} \backslash T_{v}^{\prime}$, are not in $X$, for some finite set $T_{v}^{\prime} \supset T_{v}$. The third possibility is that $\widetilde{g}$ is holomorphic on $\widetilde{l}_{v}$ and $\widetilde{g}: \widetilde{l}_{v} \rightarrow \mathbb{C}$. (Note that $\widetilde{g} \mid \widetilde{l}_{v}$ is given by the polynomial map $\psi_{v}$ which may be constant. See Theorem (1.6).) Let $K^{\prime} \subset K$ be the set of $v$ which correspond to the third case. Then

$$
\check{V}_{t}=V_{t} \cup \bigcup_{v \in K^{\prime}}\left\{\zeta_{v, 1}(t), \ldots, \zeta_{v, k(v)}(t)\right\}=\check{f}^{-1}(t), \quad t \in B^{\prime} .
$$

Let $\bar{f}:=\check{f} \circ \iota^{-1}: Y \rightarrow \mathbb{C}$. We now show how to obtain a holomorphic 1-form $\omega$ on $\check{V}_{t}$ which does not vanish on $\check{V}_{t}, t \in B^{\prime}$. Take a point $\zeta \in$ $V_{t}$. Extend $\bar{f}$ to a local function $e$ in the neighborhood of $i(\zeta) \in \mathbb{C}^{3}$ so that $e-t=h=0$ in the neighborhood of $i(\zeta)$ which is the image of the neighborhood of $\zeta \in \check{V}_{t}$ under $i$. Since $u$ and $h$ intersect transversally at $i(\zeta)$, one can define a 1 -form on $u-t=h=0$ in the neighborhood of $i(\zeta)$ as follows:

$$
d z_{1} / p_{1}=d z_{2} / p_{2}=d z_{3} / p_{3}=\omega_{1} .
$$

Here $z_{1}, z_{2}, z_{3}$ are the coordinates of $\mathbb{C}^{3}, p_{i}$ is the $2 \times 2$ determinant of partial derivatives of $u, h$ which are not with respect to $z_{i}$ (up to sign). Note that $\omega_{1}$ does not vanish. Let $\omega$ be the pullback of $\omega_{1}$ to $\check{V}_{t}$. Consider now the 1-form $\theta_{t}$ which is the restriction of $d \check{g}$ to $\check{V}_{t}$. The assumption that $F$ is a Jacobian pair yields that $\theta_{t}$ does not vanish on $V_{t}$ but does vanish on $\check{V}_{t}$. Hence the function $\phi_{t}=\theta_{t} / \omega$ gives a holomorphic function on $\check{V}_{t}$ which does not vanish on $V_{t}$ but vanishes at least at one point of $\check{V}_{t}$. Consider the 1-form $d \phi_{t} / \phi_{t}$. It is holomorphic on $V_{t}$, and it has a nontrivial residue at some point of $\breve{V}_{t}$. Hence $d \phi_{t} / \phi_{t}$ gives a nontrivial element in $\mathcal{H}^{1}\left(V_{t}\right)$. This element is invariant under the monodromy action. Contradiction to Corollary (5.2).

\section{References}

[A-C-D] E. Artal-Bartolo, P. Cassou-Noguès et A. Dimca, Sur la topologie des polynômes complexes, in: Progr. Math. 162, Birkhäuser, 1998, 317-343.

[Bai] G. Bailly-Maitre, Sur le système local de Gauss-Manin d'un polynôme de deux variables, preprint, 1998.

[B-C-W] H. Bass, E. H. Connell and D. Wright, The Jacobian Conjecture: Reduction of degree and formal expansion of the inverse, Bull. Amer. Math. Soc. 7 (1982), $287-330$. 
[B-N] S. R. Bell and R. Narasimhan, Proper holomorphic mappings of complex spaces, in: Complex Manifolds, S. R. Bell et al. (eds.), Springer, 1998, 1-38.

[Del1] P. Deligne, Equations Différentielles à Points Singuliers Réguliers, Lecture Notes in Math. 163, Springer, 1970.

[Del2] —, Théorie de Hodge, II, Publ. Math. I.H.E.S. 40 (1971), 5-58.

[Dim] A. Dimca, Invariant cycles for complex polynomials, Rev. Roumaine Math. Pures Appl. 43 (1998), 113-120.

[Dru] L. M. Drużkowski, The Jacobian conjecture: some steps towards solution, in: Automorphisms of Affine Spaces, A. van den Essen (ed.), Kluwer, 1995, $41-54$.

[Ess] A.van den Essen, Polynomial automorphisms and the Jacobian conjecture, in: Algèbre non commutative, groupes quantiques et invariants (Reims, 1995), J. Alev and G. Cauchon (eds.), Soc. Math. France, Paris, 1997, 55-81.

[F-K] H. M. Farkas and I. Kra, Riemann Surfaces, Springer, 1980.

[For1] O. Forster, Some remarks on parallelizable Stein manifolds, Bull. Amer. Math. Soc. 73 (1967), 712-716.

[For2] —, Plongements de variétés de Stein, Comment. Math. Helv. 45 (1970), 170184.

[Fri] S. Friedland, On the plane Jacobian conjecture, preprint, IHES, 1994.

[G-R] H. Grauert and R. Remmert, Coherent Analytic Sheaves, Springer, 1984.

[Gri] P. Griffiths, Periods of integrals on algebraic manifolds: summary of main results and discussion of open problems, Bull. Amer. Math. Soc. 76 (1970), 228-296.

[G-H] P. Griffiths and J. Harris, Principles of Algebraic Geometry, Wiley, 1978.

[Gro] A. Grothendieck, On the de Rham cohomology of algebraic varieties, Publ. Math. I.H.E.S. 29 (1966), 351-359.

[Hei] R. C. Heitmann, On the Jacobian conjecture, J. Pure Appl. Algebra 64 (1990), 35-72; Corrigendum, ibid. 90 (1993), 199-200.

[Kal] S. Kaliman, On the Jacobian Conjecture, Proc. Amer. Math. Soc. 117 (1993), 45-51.

[Kol] F. R. Kolchin, Differential Algebra and Algebraic Groups, Academic Press, 1973.

[K-S] T. Krasiński and S. Spodzieja, On linear differential operators related to the $n$-dimensional jacobian conjecture, in: Real Algebraic Geometry, M. Coste, L. Mahé and M.-F. Roy (eds.), Lecture Notes in Math. 1524, Springer, 1992, 308-315.

[L-W] Lê Dung Trang et C. Weber, Polynômes à fibrés rationnelles et conjecture jacobienne à 2 variables, C. R. Acad. Sci. Paris 320 (1995), 581-584.

[Lib1] A. Libgober, Alexander polynomial of plane algebraic curves and cyclic multiple planes, Duke Math. J. 49 (1982), 833-851.

[Lib2] A. Libgober, On the homotopy type of the complement to plane algebraic curves, J. Reine Angew. Math. 367 (1986), 103-114.

[Mal] B. Malgrange, Intégrales asymptotiques et monodromie, Ann. Sci. École Norm. Sup. 7 (1974), 405-430.

[Mil] J. Milnor, Singular Points of Complex Hypersurfaces, Princeton Univ. Press, 1968.

[Nag] S. Nag, The Complex Analytic Theory of Teichmüller Spaces, Wiley, 1988.

[N-S] P. Nousiainen and M. E. Sweedler, Automorphisms of polynomial and power series rings, J. Pure Appl. Algebra 29 (1983), 93-97. 
[Ore] S. Yu. Orevkov, On three-sheeted polynomial mappings of $\mathbb{C}^{2}$, Math. USSRIzv. 29 (1987), 587-596.

[Raz] M. Razar, Polynomial maps with constant jacobian, Israel J. Math. 32 (1979), 97-106.

[Sma] S. Smale, Mathematical problems for the next century, Math. Intelligencer 20 (1998), no. 2, 7-15.

[Ste1] Y. Stein, The total reducibility order of a polynomial in two variables, Israel J. Math. 68 (1989), 109-122.

[Ste2] - On the density of image of differential operators generated by polynomials, J. Anal. Math. 52 (1989), 291-300.

[Suz] M. Suzuki, Propriétés topologiques des polynômes de deux variables complexes, et automorphismes algébriques de l'espace $\mathbb{C}^{2}$, J. Math. Soc. Japan 26 (1974), 241-257.

Department of Mathematics, Statistics, and Computer Science University of Illinois-Chicago

851 S. Morgan, Chicago, IL 60607-7045, U.S.A.

E-mail: friedlan@uic.edu 